ARTICLE

DOI: $10.1038 / \mathrm{s} 41467-018-06844-4$

\title{
Src regulates amino acid-mediated mTORC1 activation by disrupting GATOR1-Rag GTPase interaction
}

Rituraj Pal ${ }^{1}$, Michela Palmieri ${ }^{1}$, Arindam Chaudhury ${ }^{2}$, Tiemo Jürgen Klisch¹, Alberto di Ronza', Joel R. Neilson², George G. Rodney ${ }^{2} \&$ Marco Sardiello ${ }^{1}$

The mechanistic target of rapamycin complex 1 (mTORC1) regulates cell survival and autophagy, and its activity is regulated by amino acid availability. Rag GTPase-GATOR1 interactions inhibit mTORC1 in the absence of amino acids, and GATOR1 release and activation of RagA/B promotes mTORC1 activity in the presence of amino acids. However, the factors that play a role in Rag-GATOR1 interaction are still poorly characterized. Here, we show that the tyrosine kinase Src is crucial for amino acid-mediated activation of mTORC1. Src acts upstream of the Rag GTPases by promoting dissociation of GATOR1 from the Rags, thereby determining mTORC1 recruitment and activation at the lysosomal surface. Accordingly, amino acid-mediated regulation of Src/mTORC1 modulates autophagy and cell size expansion. Finally, Src hyperactivation overrides amino acid signaling in the activation of mTORC1. These results shed light on the mechanisms underlying pathway dysregulation in many cancer types.

\footnotetext{
${ }^{1}$ Department of Molecular and Human Genetics, Baylor College of Medicine, Jan and Dan Duncan Neurological Research Institute, Texas Children's Hospital, Houston, TX 77030, USA. ${ }^{2}$ Department of Molecular Physiology and Biophysics, Baylor College of Medicine, One Baylor Plaza, Houston, TX 77030, USA. Correspondence and requests for materials should be addressed to M.S. (email: sardiell@bcm.edu)
} 
T he mechanistic target of rapamycin (mTOR) protein kinase is a large catalytic subunit that exists in at least two distinct complexes, mTORC1 and mTORC2, which regulate cell growth and proliferation and are often dysregulated in disease ${ }^{1-3}$. mTORC1 integrates diverse inputs, including signals associated with growth factor activity, cellular energy levels, and amino acid availability to coordinate cell metabolism ${ }^{1,2}$. Activation of mTORC1 promotes cell growth by stimulating anabolic processes such as transcription, ribosomal biogenesis and translation ${ }^{4-6}$, whereas mTORC1 inactivation promotes catabolic processes such as autophagy to meet energy demands in conditions of nutrient paucity $^{6-8}$. Many pathways that signal to mTORC1 converge on $\mathrm{TSC} 1 / 2$, a heterodimeric tumor suppressor that negatively regulates Rheb guanosine triphosphatase (Rheb GTPase), which is an essential activator of $\mathrm{mTORC1}^{2}$. Unlike many other inputs, amino acids regulate mTORC1 activity independently of TSC2 via the Rag GTPases, which form heterodimeric complexes comprised of RagA or RagB bound to RagC or $\operatorname{RagD}^{9}$.

Amino acid signaling to mTORC1 requires a lysosomal membrane-associated machinery that consists of the Rag GTPases, the Ragulator complex, and the vacuolar adenosine triphosphatase (V-ATPase) $)^{10,11}$. In response to amino acids, the guanine nucleotide exchange factor (GEF) activity of Ragulator is promoted toward RagA and RagB that, when guanosine triphosphate (GTP)-loaded, recruit mTORC1 to the lysosomal surface. mTORC1 may then be fully activated by its potent and essential direct activator, Rheb ${ }^{12,13}$. Upon removal of amino acids, Rag GTPase-activating protein (GAP) complex GATOR1 induces the Rag dimers to switch into an inactive conformation containing guanosine diphosphate (GDP)-bound $\operatorname{Rag} \mathrm{A} / \mathrm{B}$, thereby releasing mTORC1 from the lysosomal surface, which in turn results in the inactivation of $\mathrm{mTORC}^{10}$. Upon addition of amino acids, GATOR2, a positive regulator of the Rags, initiates GTP loading of $\operatorname{RagA} / \mathrm{B}$ via inhibition of GATOR1 $1^{10,14,15}$. Amino acid stimulation promotes dissociation of GATOR1 from the Rags ${ }^{9}$, thus establishing GATOR1 repression of the Rags as a major regulatory axis of mTORC1 activation in response to amino acids.

Owing to the central role of mTORC1 in the control of cell growth and metabolism, numerous studies have investigated regulation of mTORC1 by oncogenes and tumor suppressors that are implicated in familial or sporadic forms of cancer ${ }^{16,17}$. Among these, the non-receptor tyrosine kinase, Src, is an oncogene of paramount importance. Several studies have identified Src as a critical component of the signal transduction pathways that control cancer cell growth ${ }^{18,19}$.

Here, we show that Src is a critical regulator of amino acidmediated activation of mTORC1. We demonstrate that Src acts upstream of the Rag GTPases by promoting dissociation of GATOR1 from the Rags, thereby modulating mTORC1 recruitment and activation at the lysosomal surface. Accordingly, we show that amino acid-mediated regulation of Src/mTORC1 modulates autophagy and cell size expansion. In addition, we show that Src hyperactivation overrides amino acid signaling in the activation of mTORC1, a finding that could shed light on the mechanisms underlying pathway dysregulation in many cancer types.

\section{Results}

Src regulates amino acid-mediated mTORC1 activation. We hypothesized that Src is involved in amino acid-mediated regulation of mTORC1, and set out to test this hypothesis by first defining the conditions in which optimal stimulation of mTORC1 by amino acids is observed in cultured SH-SY5Y cells and mouse embryonic fibroblasts (MEFs). A time-course analysis showed that consistent activation of mTORC1 occurs $30 \mathrm{~min}$ after amino acid replenishment in both cell lines (Supplementary Fig. 1a,b). We therefore used a 30-min period of amino acid stimulation in subsequent studies. Consistent with previous reports ${ }^{12}$, we found that, in SH-SY5Y cells, mTOR predominantly co-localized with LAMP2-positive lysosomal vesicles upon amino acid stimulation, whereas amino acid starvation promoted cytosolic localization of mTOR with minimal amounts on the lysosomal surface (Supplementary Fig. 1c). To investigate whether amino acid activation of mTORC1 depends on Src function, we suppressed Src activity by using either short hairpin RNAs (shRNAs) targeting $S R C$, or Src pharmacological inhibitors (dasatinib ${ }^{20}$ and $\mathrm{PP}^{21}$ ). Both genetic and pharmacological inhibition of Src activity decreased amino acid-induced activation of mTORC1 with no changes in total mTOR protein levels (Fig. 1a, b and Supplementary Fig. 1df). While PP2 treatment suppressed amino acid-mediated activation of both Src and mTORC1, rapamycin only inhibited mTORC1 activity (Fig. 1c), indicating that Src is an upstream regulator of mTORC1. Confocal microscopy experiments showed that mTOR translocation from the cytosol to lysosomes upon amino acid stimulation was abolished by pharmacological or genetic inhibition of Src activity (Fig. 1d, e). Thus, Src acts as an essential upstream factor that controls amino acid-dependent localization and activation of mTORC1.

We next tested whether Src activity per se is sufficient for the activation of mTORC1, irrespective of amino acid availability. From the $\mathrm{N}$-terminus to $\mathrm{C}$-terminus, $\mathrm{Src}$ is comprised of a myristoyl group attached to a unique SH4 domain (U) followed by $\mathrm{SH} 3$ and $\mathrm{SH} 2$ domains, an $\mathrm{SH} 2$-kinase linker, a protein-tyrosine kinase domain (also known as SH1 domain), and a C-terminal regulatory segment ${ }^{19,22}$. Mutations at Src Y530 or deletion of $\mathrm{Src} \mathrm{SH} 2 / \mathrm{SH} 3$ domains result in constitutive activation of $\mathrm{Src}^{23}$ as evidenced by autophosphorylation at Y419 22 (Supplementary Fig. 1g,h). Wild-type Src (WT-Src-Flag) and constitutively active Src constructs obtained by mutating Y530 to phenylalanine (Y530F-Src) ${ }^{24,25}$ or by deleting the $\mathrm{SH} 3$ or $\mathrm{SH} 2$ domain ( $\mathrm{Src} \Delta \mathrm{SH} 3$-Flag and $\mathrm{Src} \Delta \mathrm{SH} 2$-Flag) were expressed in cultured cells. All constructs significantly increased mTORC1 activity in normal growth conditions, which was attenuated by PP2-mediated Src inhibition (Supplementary Fig. 1i, j). Interestingly, transfection of constitutively active Src resulted in insensitivity of mTORC1 to amino acid deprivation, which was reversed by pharmacological inhibition of Src (Fig. 1f, g). These results suggest that the kinase activity of Src is both necessary and sufficient to induce mTORC1 activation in the context of the amino acid-sensing machinery.

Src regulates mTORC1 at lysosomes independently of TSC2. To investigate whether Src-dependent amino acid regulation of mTORC1 funnels through TSC2, a known key negative regulator of mTORC1 activity ${ }^{2}$, we used Tsc2 $2^{-1-}$ MEFs (Fig. 2a) where the activity of mTORC1 is significantly higher than in WT MEFs $\left(T s 2^{+/+}\right)$independent of amino acid availability. Growth factors such as insulin regulate mTORC1 activity through the Akt-TSC2 axis $^{2}$. While in Tsc2 $2^{+/+}$MEFs insulin-mediated stimulation of mTORC1 activity was abolished upon treatment with the Akt inhibitor MK2206 26 , Tsc2 ${ }^{-1-}$ MEFs were insensitive to both insulin deprivation and Akt inhibition (Supplementary Fig. 2a). Insulin regulation of mTORC1 signaling was independent of Src activity (Supplementary Fig. 2b) while amino acid stimulation of mTORC1 was independent of Akt activity (Supplementary Fig. 2c). Activation of mTORC1 was further potentiated post amino acid stimulation (Fig. 2b), but this was attenuated by inhibition of Src in both $T s c 2^{-/-}$and $T s c 2^{+/+}$cells (Fig. 2c). mTORC1 localization at the lysosomal surface in response to amino acids was independent of TSC2 presence; however, 
a
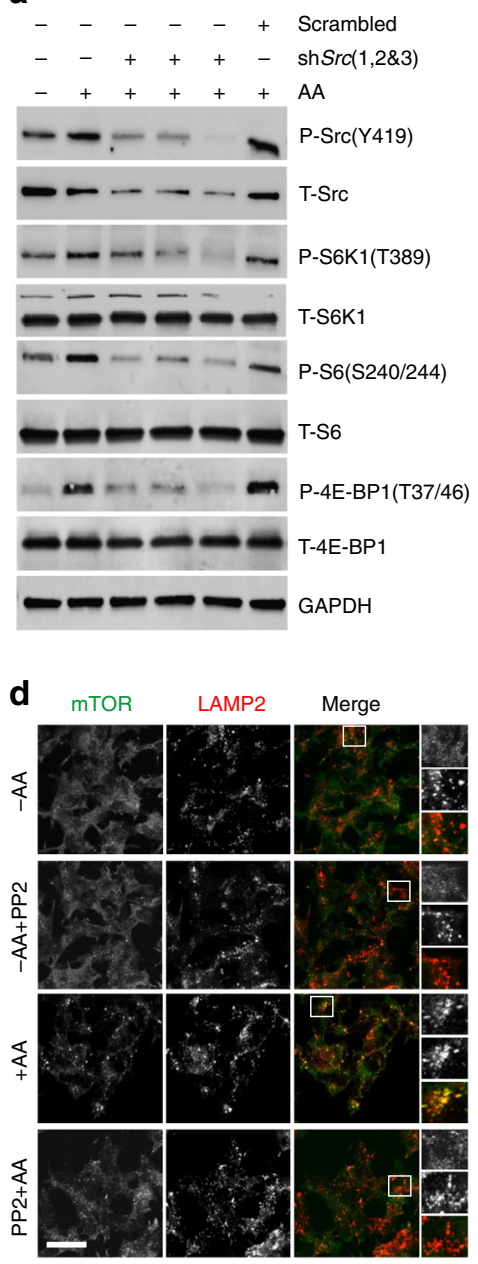

f

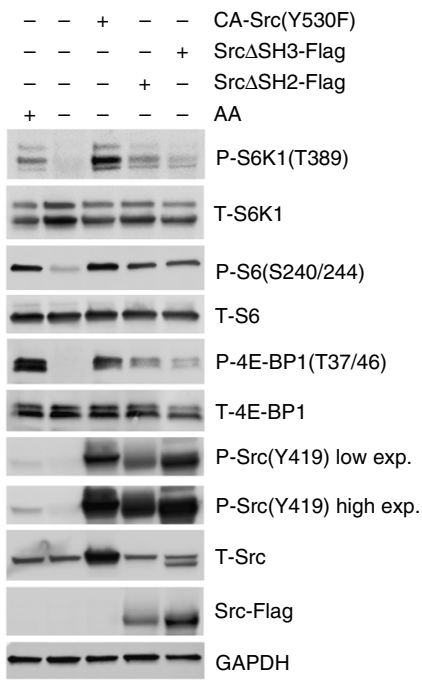

b

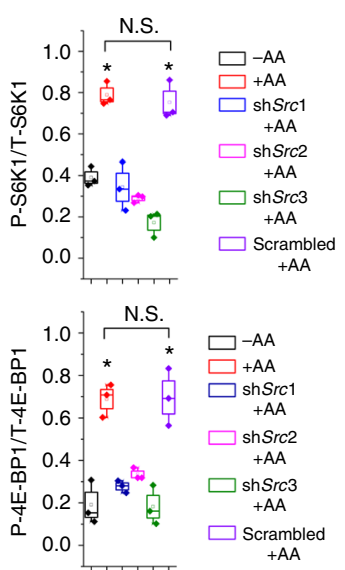

$\begin{array}{llllllllll}0 & 5 & 15 & 30 & 75 & 5 & 15 & 30 & 75 & \text { Time }(\mathrm{min})\end{array}$

$-\quad-\quad-++++\operatorname{PP} 2$

$+++++++++\mathrm{AA}$

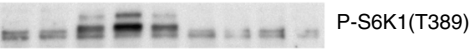

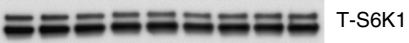

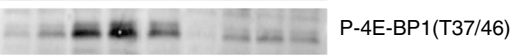

-

- - - - - - P-S6(S240/244)

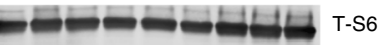

$=0=0 \mathrm{GAPDH}$

e
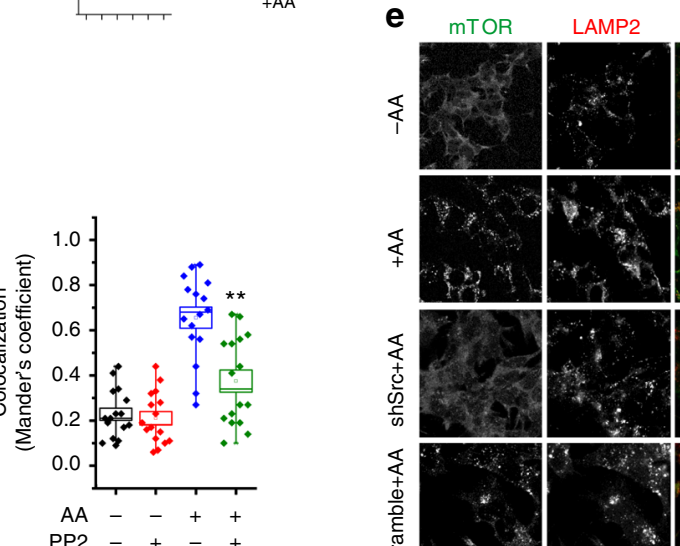

Merge
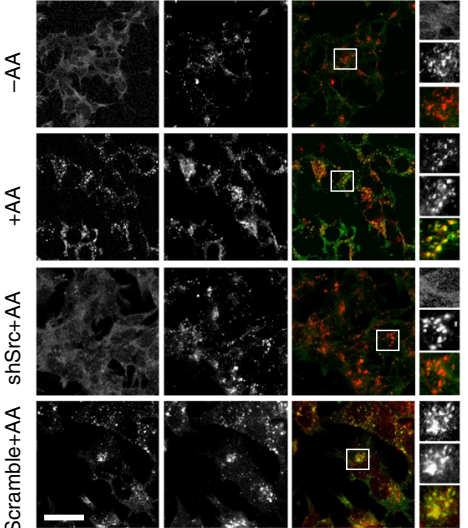

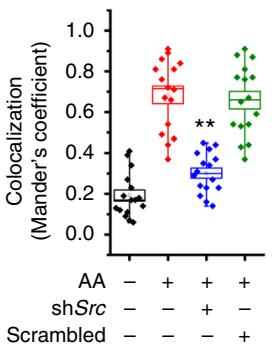

。

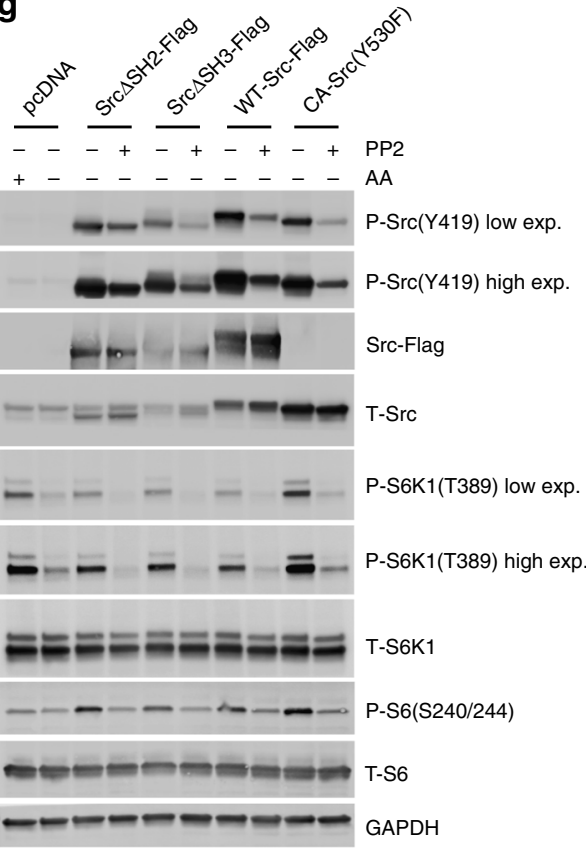

C

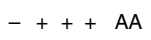

-++ Rapamycin

- -+ + PP2

- - P-Src(Y419)

- $=-\mathrm{T}-\mathrm{Src}$

$==-\mathrm{P}-\mathrm{S} 6 \mathrm{~K} 1$ (T389)

=ニニ= T-S6K1

- - P-S6(S240/244)

- T-S6

$-\square-$ GAPDH

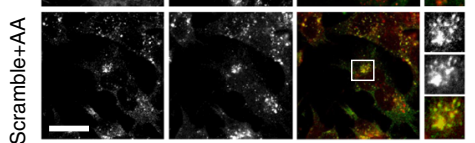

Scrambled -
mTORC1 translocation to lysosomes was decreased by PP2mediated inhibition of Src in both $T s c 2^{+/+}$and $T s c 2^{-1-}$ cells (Supplementary Fig. 2d, e) while, as expected, mTORC1 localization was independent of Akt activity. Together, these results establish that Src-dependent amino acid regulation of mTORC1 is independent of TSC2 signaling.
Next, we investigated the mechanism of mTORC1 activation by $\mathrm{Src}$ in response to amino acids. Rag GTPases are essential for the recruitment of $\mathrm{mTORC} 1$ at the lysosomal surface in response to amino acids. Previous reports have demonstrated that knockdown of the Rags releases mTORC1 from the lysosomal surface, thereby inactivating mTORC1 independently of amino 
Fig. $1 \mathrm{Src}$ is necessary and sufficient for amino acid-mediated activation of mTORC1. a SH-SY5Y cells were treated with SRC shRNAs, starved, and stimulated with amino acids for $30 \mathrm{~min}$. Lysates were probed with antibodies as indicated. Box plots represent SE of $n=3$ independent experiments. ${ }^{\star} p<$ 0.05. N.S. represents "not significant". b SH-SY5Y cells were starved and treated with vehicle (DMSO) or PP2 (10 $\mu$ M) for the last $2 \mathrm{~h}$ of starvation prior to stimulation with amino acids at several time points. Lysates were probed with antibodies as indicated. c SH-SY5Y cells were starved and treated with vehicle (DMSO) or PP2 $(10 \mu \mathrm{M})$, or rapamycin $(600 \mathrm{nM})$ for the last $2 \mathrm{~h}$ of starvation and then stimulated with amino acids (30 min). Lysates were probed with antibodies as indicated. $\mathbf{d}$ SH-SY5Y cells were treated as in $\mathbf{b}$ but with a single 30 min amino acid stimulation prior to immunofluorescence labeling of endogenous LAMP2 (red) and mTOR (green). e SH-SY5Y cells were treated as in a and labeled and analyzed as in d. Bar indicates $60 \mu \mathrm{m}$. Representative cells are shown where yellow or orange pixels indicate co-localization in the merged images. In all images in $\mathbf{d}$ and $\mathbf{e}$ insets show selected fields that were magnified by a factor of 4 . Scale bar indicates $60 \mu \mathrm{m}$. Quantified data represent mean co-localization (Mander's coefficient) of mTOR and LAMP2 in at least 15 cells for each condition. Box plots represent SE of $n=3$ independent experiments. ${ }^{\star \star}$ indicates significant differences ( $\left.{ }^{\star \star} p<0.01\right)$ between all conditions in each group. $\mathbf{f} \mathrm{SH}-\mathrm{SY} 5 \mathrm{Y}$ cells, transiently transfected with Y530F-Src, Src $\Delta \mathrm{SH}$, or $\mathrm{Src} \Delta \mathrm{SH} 2$, were starved with amino acids (kept in dialyzed serum for $4 \mathrm{~h}$ ). Cells were then lysed and immunoblot analyses were used to measure the levels of the indicated proteins and phosphorylation states. $\mathbf{g} \mathrm{SH}-$ SY5Y cells were transiently transfected with the indicated Src constructs and starved as in $\mathbf{f}$. Cells were treated with PP2 (10 $\mu \mathrm{M})$, where indicated, for the last $2 \mathrm{~h}$ of starvation prior to lysis and immunoblot analyses. GAPDH was used as a loading control in all immunoblot assays. Statistical differences between groups in $\mathbf{a}, \mathbf{d}$, and $\mathbf{e}$ were determined using ANOVA with Tukey's post hoc test

acid availability ${ }^{12,27}$. We performed cell fractionation experiments and observed that Src is enriched with heavy membranes, similar to the lysosomal membrane proteins LAMP1 and LAMP2; gradient fractionation confirmed the presence of $\mathrm{Src}$ in the lysosome-enriched fraction (Fig. 2d, Supplementary Fig. 2f, g) with low amounts of Src in the cytosol. Confocal analysis showed that Src associates with lysosomes and RagC, independently of amino acid availability (Fig. 2e, f). We did not detect any obvious association of Src with other membrane-bound organelles such as the Golgi complex, mitochondria, peroxisomes, and the endoplasmic reticulum (Supplementary Fig. 2h). In addition, co-IP experiments showed that HA-GST-RagA/C interact with Src (Fig. 2g). Co-IP experiments confirmed that endogenous Src interacts with endogenous RagA and RagC (Fig. 2h) and that these interactions are independent of amino acid availability (Fig. 2i).

Src promotes recruitment of mTORC1 at the lysosomal surface. We then investigated whether Src promotes mTORC1 recruitment at the lysosomal surface by regulating the GTP/GDPbound state of the Rag GTPases in response to amino acids. We confirmed that Src regulates mTORC1 localization and activity at the lysosomal surface in response to amino acids in HEK293 cells (Supplementary Fig. 3a, b). Co-IP analysis in HEK293 cells showed that shRNA-mediated knockdown of SRC decreased the interaction of RagA with raptor, a component of mTORC1 complex $^{12}$ (Fig. 3a). As a control, RagC interaction with RagA was not affected by knockdown of SRC. The specificity of the interaction between Rag and raptor was confirmed by IgG co-IP assay (Supplementary Fig. 3c). Co-IP analysis in HEK293 cells showed significantly stronger interactions of raptor and mTOR with the Rags in amino acid-stimulated cells compared to amino acid-depleted cells, which was suppressed by PP2 treatment (Fig. 3b, c and Supplementary Fig. 3d). mTORC1 activity was consistent with the status of the Rag-mTOR interaction (Fig. 3a, b). To determine whether Src regulates Rag-raptor interaction through the GTP/GDP loading status of the Rags, we used Rag nucleotide binding mutants that are either constitutively GTPbound and therefore active (HA-GST-RagA GTP: Q66L) ${ }^{27}$ or that bind negligible amounts of nucleotides and are therefore inactive (HA-GST-RagCGDP: S75N) ${ }^{27}$. Co-IP experiments in cells stably expressing Flag-raptor showed that amino acid-mediated increases in both Rag-raptor and Rag-mTOR interactions were abolished by Src inhibition in cells expressing WT RagA/C, whereas both interactions became resistant to amino acid starvation or Src inhibition in cells expressing RagA ${ }^{\mathrm{GTP}} / \mathrm{RagC}^{\mathrm{GDP}}$
(Fig. 3d), thus suggesting that Src promotes Rag-mTOR interaction by regulating the GDP/GTP-loading status of the Rags.

Given that the Rag GTPases are responsible for the subcellular localization of $\mathrm{mTORC}^{12}$ and Src regulates the Rag GTPases, we asked whether the observed effects of Src activity on mTOR localization depend on the Rags. Confocal analysis of cells expressing WT RagA/C showed that, upon amino acid deprivation, mTOR distributed in tiny puncta throughout the cytoplasm, whereas it localized to large vesicular structures in the perinuclear region in response to amino acid stimulation (Fig. 3e). In cells expressing constitutively active RagA/C ( $\operatorname{RagA}{ }^{G T P} / \operatorname{RagC}^{\mathrm{GDP}}$ ), mTOR localized to the perinuclear vesicles independent of the availability of amino acids (Fig. 3f). PP2 was unable to alter the localization of mTOR in the presence of constitutively active $\mathrm{RagA}^{\mathrm{GTP}} / \mathrm{RagC} \mathrm{GDP}^{\mathrm{G}}$. Amino acid-mediated stimulation of mTORC1 activity was abolished by Src inhibition in cells expressing WT RagA/C, whereas mTORC1 activity became resistant to amino acid starvation or Src inhibition in cells expressing RagAGTP/RagCGDP (Fig. 3g). Similar results were obtained in SHSY-5Y cells (Supplementary Fig. 3e). Together, these findings establish that Src regulates amino acid-mediated stimulation of mTORC1 activity via the Rag GTPases.

Src disrupts GATOR1-Rag GTPase interaction. A recent report identified GATOR1, a complex comprised of Depdc5, Nprl2, and $\mathrm{Nprl3}$, as a negative regulator of mTORC1 that acts by increasing GTP hydrolysis of RagA and RagB ${ }^{9}$. Loss of GATOR1 function renders mTORC1 signaling insensitive to amino acid starvation ${ }^{9}$. Rag-GATOR1 interaction is decreased by amino acid stimulation ${ }^{9}$ and is independent of the availability of growth factors (Supplementary Fig. 4a). Since Src activation of mTORC1 is dependent on the GTP/GDP loading status of the Rags, we hypothesized that Src activation results in the disruption of GATOR1-Rag interaction. Co-IP assays in HEK293 cells transiently transfected with GFPDepdc5 showed that Src interacts with GATOR1 (Fig. 4a). Consistent with a previous study ${ }^{9}$, we found that shRNA-mediated knockdown of GATOR1 (Supplementary Fig. 4b) decreased HEK cell size (Supplementary Fig. 4c) and blocked the inactivation of mTORC1 that is normally caused by amino acid deprivation (Supplementary Fig. 4d). To test whether Src regulation of mTORC1 activity is mediated through GATOR1, we knocked down GATOR1 by using shRNAs targeting DEPDC5, NPRL2, and NPRL3 genes. While confirming that loss of GATOR1 makes mTORC1 insensitive to amino acid starvation, our results show that loss of GATOR1 makes mTORC1 activity resistant to Src inhibition (Fig. 4b), thus indicating that Src regulates mTORC1 via GATOR1. Next, we tested whether Src activates 
a

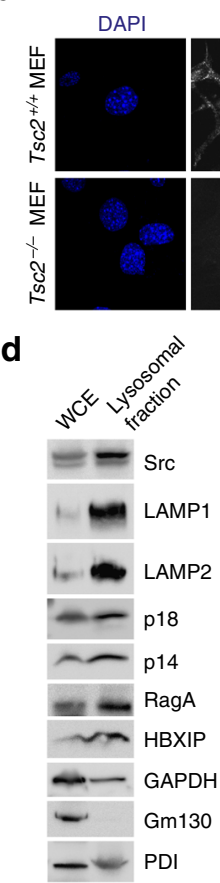

b
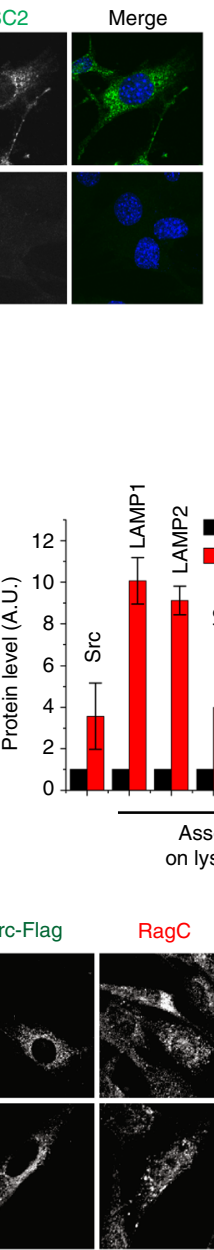

$\mathbf{g}$

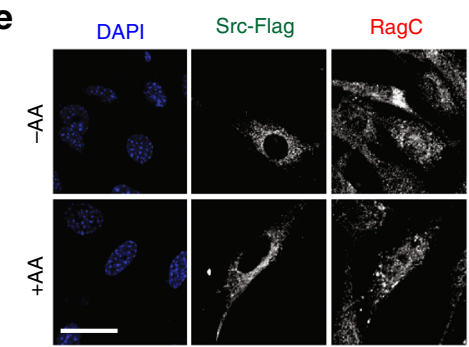

$+\quad+\quad-\quad \mathrm{IP}: \lg \mathrm{G}$

$-\quad++$ IP: Src

-+++ HA-GST-RagC

- + + + HA-GST-RagA
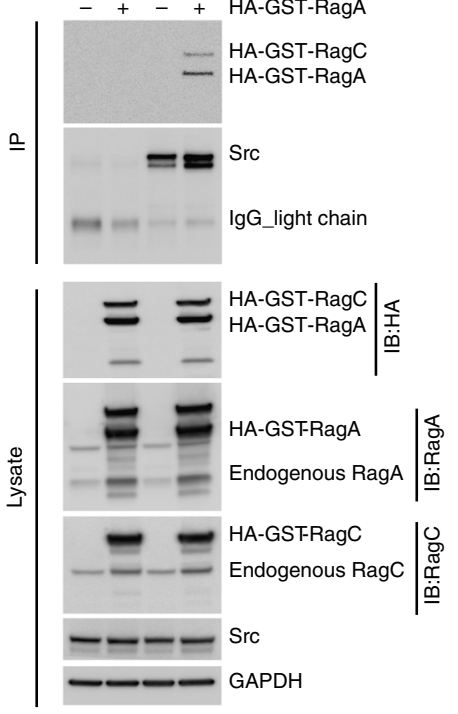

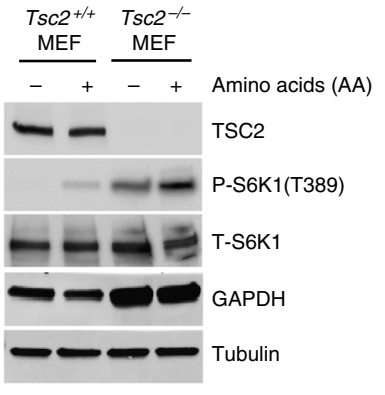

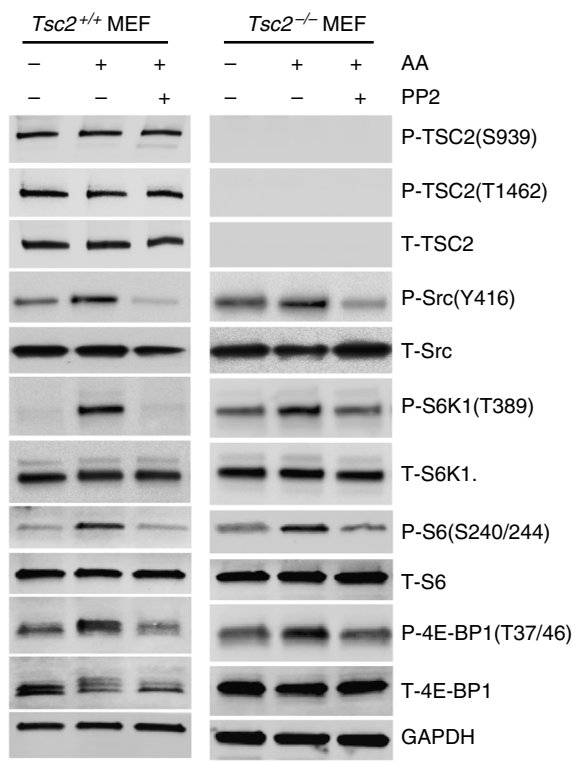

Associate
on lysosome not associate

$\mathbf{f}$

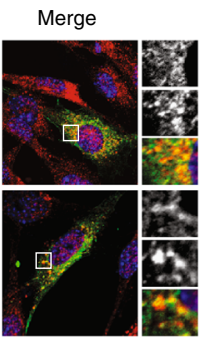

h

$+\quad-\quad I P: \lg G$

- + - IP: RagA

- + + IP: RagC
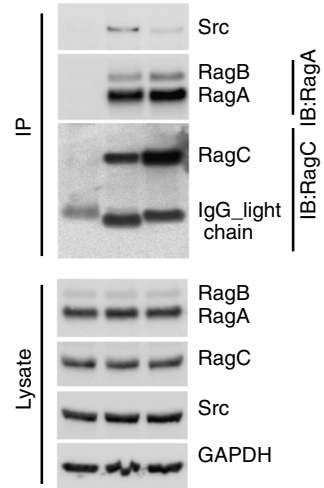

DAPI
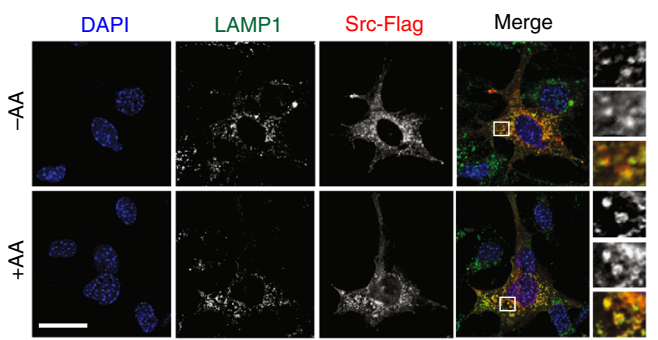

|

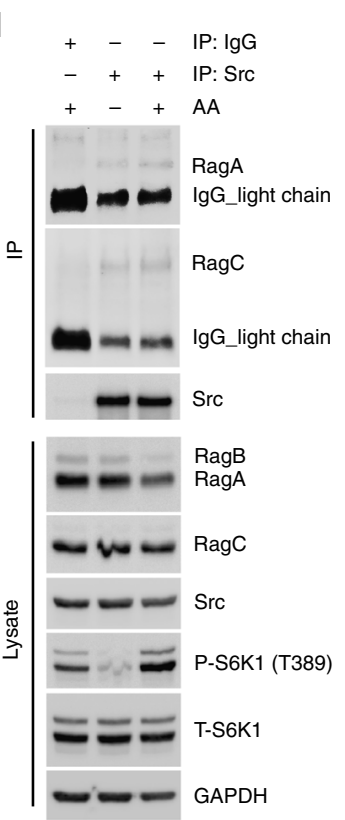

Rag-mTORC1 signaling by dissociating GATOR1 from Rag proteins. Expression of constitutively active Src (CA-Src) dramatically reduced GATOR1-Rag interaction (Fig. 4c); as a consequence, a strong activation of mTORC1 was observed. Importantly, expression of a kinase-dead Src construct (KD-Src/K295R-Src) did not alter Rag-GATOR1 interaction (Fig. 4d). Expression of WTSrc also decreased Rag-GATOR1 interaction (Fig. 4e), which was reversed by inhibition of Src with PP2 (Fig. 4f). Together, these 
Fig. 2 Src regulates mTORC1 activity independently of TSC2. a WT (Tsc2 ${ }^{+/+}$) and TSC2 null (Tsc2 ${ }^{-/-}$) MEF cells were coimmunostained for DAPI (blue) and TSC2 (green). Bar indicates $40 \mu \mathrm{m}$. b Amino acid-starved Tsc2 ${ }^{-/-}$MEFs showed significantly higher activity of mTORC1 compared to Tsc2 ${ }^{+/+}$MEFs. Activation was further potentiated upon stimulation with amino acids. Lysates were probed with antibodies as indicated. $\mathbf{c} T s c 2^{+/+}$and $T s c 2^{-/-} \mathrm{MEFs}$ cells were starved and treated with vehicle (DMSO) or PP2 $(10 \mu \mathrm{M})$ for the last $2 \mathrm{~h}$ of starvation and then stimulated with amino acids (30 min). Lysates were probed with antibodies as indicated. $\mathbf{d}$ A lysosome-enriched cell fraction was isolated from SH-SY5Y cells and used to perform immunoblot analyses to measure the levels of the indicated proteins. LAMP1, LAMP2, p18, p14, RagA, and HBXIP are known lysosome-associated protein ${ }^{10}$. GM130 and PDI are used as Golgi and ER markers, respectively 47,48 . WCE stands for whole-cell extract. Quantified data represent means \pm SEM of $n=2-3$ independent experiments. e, $\mathbf{f} \mathrm{MEF}$ cells were transfected to express Src, starved and stimulated with amino acids (30 min) prior to immunofluorescent labeling of RagC (red) and Src (green) in e and LAMP1 (green) and Src (red) in f. Representative cells are shown where yellow or orange pixels indicate co-localization in the merged images. In all images, insets show selected fields that were magnified by a factor of 4 . Bar indicates $40 \mu \mathrm{m}$. $\mathbf{g} \mathrm{HEK}$ cells, transiently transfected with HA-GST-RagA and HA-GST-RagC, were lysed and co-IP analyses were performed to test interaction of Src with the Rags. Immunoblot analyses were used to measure the levels of the indicated proteins. $\mathbf{h}$ HEK cells were lysed and colP analyses were performed to test interaction of Src with the Rags. i HEK cells were starved prior to amino acid stimulation (30 min). Cells were lysed and colP analyses were performed. IgG antibody pulldown was performed as a negative control in $\mathbf{g}$ to $\mathbf{i}$. GAPDH and tubulin were used as a loading control in immunoblot assays

results demonstrate that Src kinase regulates mTORC1 activity by controlling GATOR1-Rag interaction.

Increased mTORC1 activity in cells with high Src activity. Given that Src positively regulates mTORC1 activity, we asked whether mTORC1 activity is higher in cells having endogenous activation of Src. To test this possibility, we investigated Src-mTORC1 signaling in HT-29 and Caco-2 colon cancer cells which have higher Src activity compared to normal colon cells ${ }^{28-31}$. We observed a significant increase in $\mathrm{mTORC} 1$ activity in both Caco-2 and HT-29 cells compared to the normal colon epithelial cell line $\mathrm{FHC}^{28}$ under amino acid starvation (Fig. 5a, b), similar to cells transfected with active Src constructs (Fig. 1g). Confocal microscopy experiments showed that amino acid stimulation increased mTOR translocation from the cytosol to lysosomes in FHC, Caco-2, and HT-29 cells (Fig. 5c); however, lysosomal localization of mTOR was significantly higher in Caco2 and HT-29 cells compared to FHC cells under amino acid starvation (Fig. 5d). Interestingly, inhibition of Src abolished mTORC1 activity under both amino acid starvation and stimulation in Caco-2 cells (Fig. 5e), suggesting that increased activation of endogenous Src leads to higher mTORC1 activity as compared to cells with normal Src activity.

Src regulates cell growth, TFEB localization, and autophagy. Next, we investigated the role of Src in mTORC1 regulation of cell growth and autophagy. Prolonged cell exposure to PP2 significantly decreases the size of SH-SY5Y cells (Fig. 6a), MEFs (Fig. 6b), and HEK293 cells (Supplementary Fig. 5a), thus indicating that Src activity is necessary to sustain cell growth. Live imaging of SH-SY5Y cells transfected with GFP-LC3, a wellestablished probe to monitor autophagy induction ${ }^{32,33}$, showed that amino acid stimulation results in a decrease in LC3-positive autophagic vesicles compared to starved cells (Fig. 6c). Src inhibition, however, blocked amino acid-induced inhibition of autophagosome formation similar to the mTORC1 inhibitor rapamycin (Fig. 6c) ${ }^{34}$. Consistently, live imaging of HEK cells transfected with GFP-LC3 showed a significant reduction in LC3positive autophagic vesicles in cells transfected with an shRNA targeting $S R C$ (Supplementary Fig. 5b), thus indicating a critical role of Src in mTORC1 regulation of autophagy in response to amino acids. To test whether the observed increase in the number of autophagosomes was the result of an increase in the synthesis and not in the accumulation of the autophagosomes due to impairment of autophagic flux, we transfected cells with a tandem GFP-RFP-LC3 construct that emits a far-red fluorescent signal when autophagosomes fuse with lysosomes, and a yellow fluorescent signal when autophagosomes are unable to fuse with lysosomes ${ }^{35,36}$. A prevalence of red vesicles confirmed that LC3IIpositive vesicles fused with lysosomes in the presence of PP2 (Supplementary Fig. 5c), indicating a functionally active autophagic system. Consistent with these results, immunoblot assays showed an increase in the amount of LC3II and a concomitant decrease in P62 level upon knockdown of SRC (Supplementary Fig. 5d). In addition, amino acid-mediated decrease in the amounts of LC3II was significantly reversed upon knockdown of $S R C$ (Fig. 6d). Consistently, a reduction in the amounts of LC3II and a concomitant increase in mTORC1 activity were observed upon amino acid stimulation and reversed by Src inhibition (Fig. 6e). Similar results were obtained in HEK293 cells (Supplementary Fig. 5e).

mTORC1 regulates the expression of autophagy and lysosomal genes by controlling the activity of the transcription factor EB (TFEB), a master regulator of autophagy and lysosomal genes ${ }^{26,37-39}$. TFEB phosphorylation by mTORC1 promotes TFEB retention in the cytosol, thus hampering its positive regulation of transcription ${ }^{40}$. Amino acid signaling to mTORC1 correlates with TFEB subcellular localization ${ }^{41}$. We tested whether Src regulation of mTORC1 activity modulates the localization of TFEB in response to amino acids. Confocal microscopy showed that amino acid starvation increases nuclear translocation of TFEB, whereas amino acid stimulation blocked nuclear translocation of TFEB (Fig. $6 \mathrm{~g}$ ), as previously reported ${ }^{42}$. ShRNA-mediated knockdown of SRC promoted TFEB nuclear translocation in the presence of amino acids (Supplementary Fig. $5 \mathrm{f}$ and Fig. 6f). Consistently, pharmacological inhibition of Src showed a significant increase in nuclear TFEB similar to Torin1 (Fig. 6g), a catalytic inhibitor of mTOR $^{43}$. TFEB is known to localize to lysosomes in an amino acid-dependent manner via recruitment by active $\mathrm{Rags}^{42}$. In cells stimulated with amino acids, TFEB showed an increased association with lysosomes (Fig. 6h). Strikingly, pretreatment with PP2-which as shown above causes inhibition of both Rag and mTORC1 activityresulted in a substantial translocation of TFEB to the nucleus, with no detectable association of TFEB with lysosomes. In contrast, pretreatment with Torin1 resulted in a dual localization of TFEB to the nucleus and to lysosomes, consistent with the notion that Torin 1 inhibits mTORC1 activity but not amino aciddependent activation of the Rags. Collectively, these results demonstrate that Src controls the activity of the Rag GTPases and mTORC1 and the subcellular localization of TFEB in response to amino acids, thereby regulating autophagy and cell growth.

\section{Discussion}

Results from this study define Src as an essential upstream positive regulator of amino acid-induced mTORC1 signaling 
a

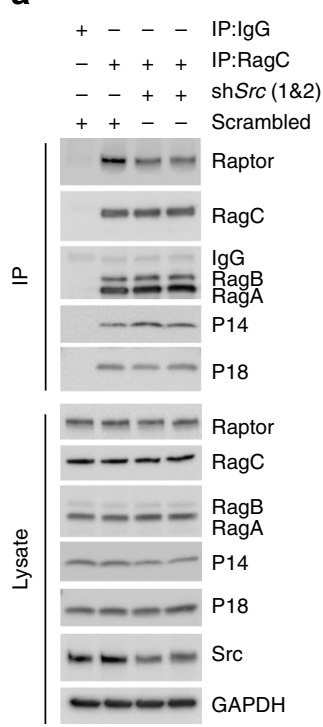

b

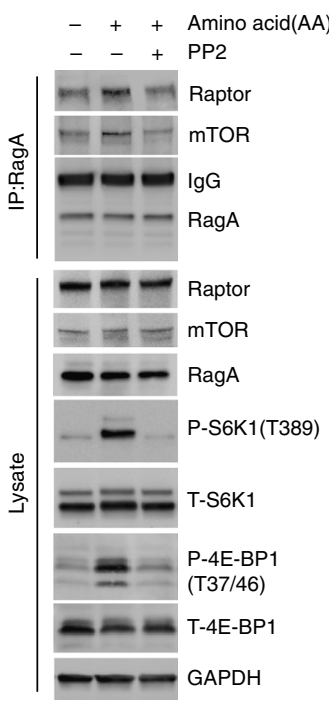

C

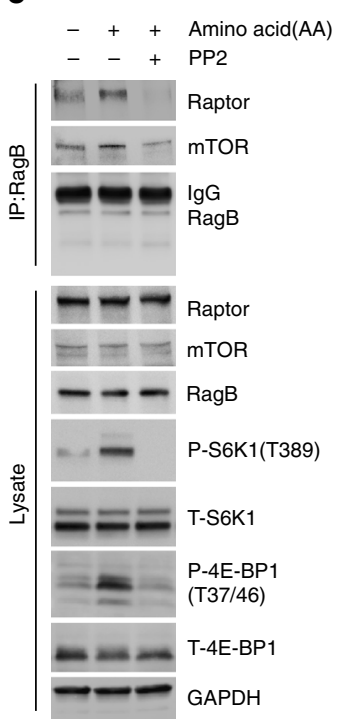

d

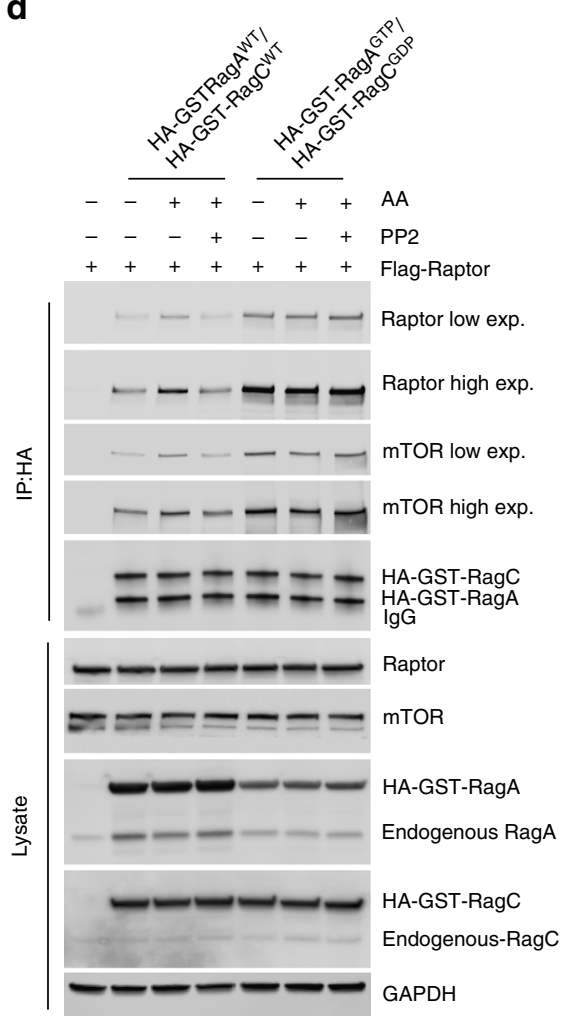

e

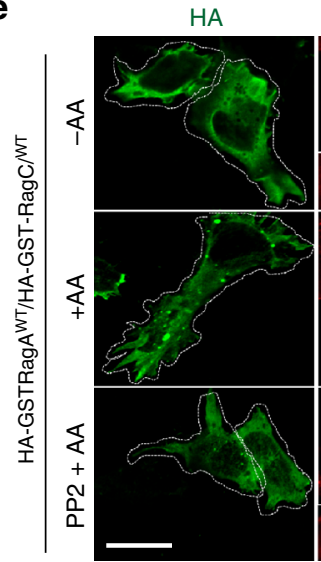

f

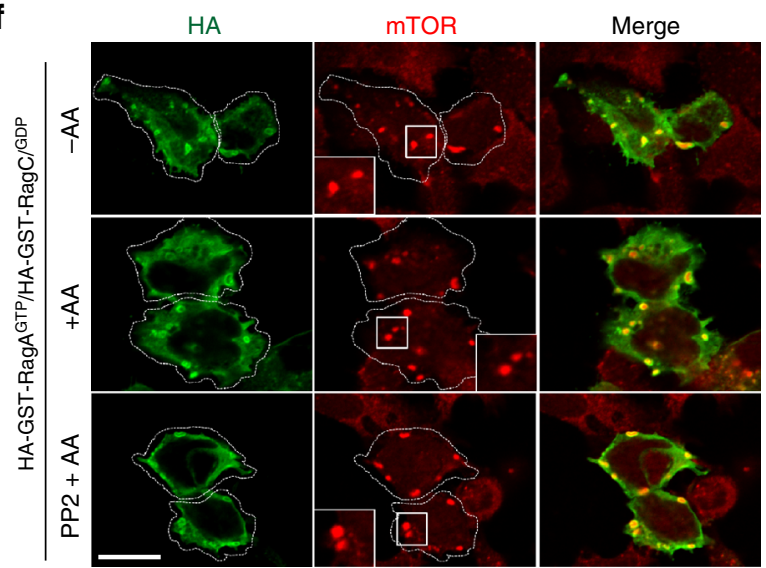

mTOR Merge

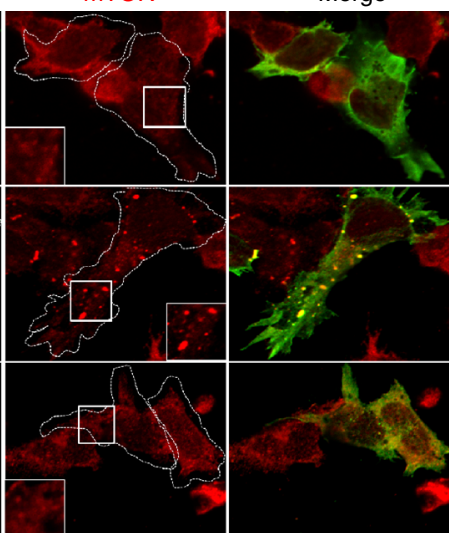

mTOR
9
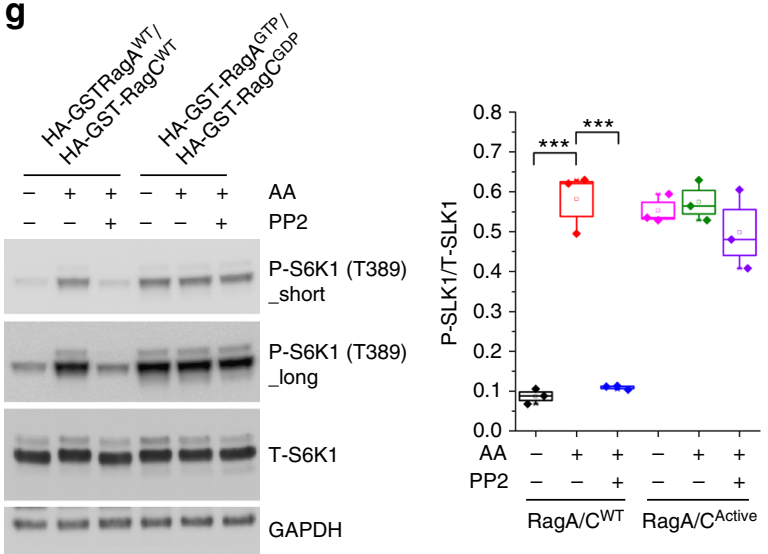

(Fig. 7). Src regulates localization and activation of mTORC1 upon amino acid stimulation, and Src activity per se is sufficient for the activation of mTORC1, irrespective of amino acid availability. Our work also leads to a number of novel questions which will be interesting to explore in future studies. First, what is the upstream mechanism that activates Src in response to amino acids? Sestrin 2 and CASTOR1 were characterized as sensors for cytosolic leucine and arginine, respectively ${ }^{44-46}$. Upon amino acid binding to sestrin2 or CASTOR1, GATOR2 dissociates from them and initiates GTP-loading of $\operatorname{RagA} / \mathrm{B}$ via inhibition of GATOR $1^{14,15}$. It is possible that GATOR2 transfers the amino acid signal to Src, which then initiates GTP-loading of RagA/B by dissociating Rag-GATOR1 interaction. GATOR2, once released from sestrin2 or CASTOR1, might interact with Src and lead to a conformational change that activates Src thus enabling dissociation of GATOR1 from the Rags. Future studies that investigate the molecular association between GATOR2 and Src could establish whether this mechanism funnels so far unidentified 
Fig. 3 Src promotes mTORC1 recruitment to lysosomes. a HEK cells transiently transfected with scrambled shRNA or shSrc prior to colP analyses. Lysates were used for immunoprecipitation by either Src or lgG antibody (negative control). b, c HEK293 cells were starved and treated with vehicle (DMSO) or PP2 $(10 \mu \mathrm{M})$ for the last $2 \mathrm{~h}$ of starvation and then stimulated with amino acids ( $30 \mathrm{~min}$ ). colP analyses were performed to test interaction of mTOR and Raptor with RagA in b or RagB in c. Immunoblot analyses were used to measure the levels of the indicated proteins and phosphorylation states. $\mathbf{d}$ HEK293 cells stably expressing Flag-raptor, transiently transfected with HA-GST-RagAWT/CWT or HA-GST-RagA GTP/CGDP, were starved and treated with vehicle (DMSO) or PP2 $(10 \mu \mathrm{M})$ for the last $2 \mathrm{~h}$ of starvation and then stimulated with amino acids ( $30 \mathrm{~min})$. colP analyses were performed to test interaction of mTOR and Raptor with RagA/C. Immunoblot analyses were used to measure the levels of the indicated proteins. e, f HEK293 cells were transiently transfected with HA-GST-RagAWT/CWT (e) or HA-GST-RagAGTP/CGDP (f) and treated as in a prior to immunofluorescence labeling of HA (green) and endogenous mTOR (red). Representative cells are shown where punctate structures indicate lysosomal localization of mTOR. Magnified images are represented in white boxes. Bar indicates $40 \mu \mathrm{m}$. $\mathbf{g}$ HEK293 cells stably expressing HA-GST-RagAWT/CWT or HA-GST-RagA GTP/CGDP were treated as in b. Immunoblot analyses were used to measure the levels of the indicated proteins and phosphorylation states. Short and long indicate short and long exposure, respectively. Box plots represent SE of $n=3$ independent experiments. ${ }^{\star \star \star} p<0.001$. Statistical differences between groups were determined using ANOVA with Tukey's post hoc test. GAPDH was used as a loading control in all immunoblot assays

regulatory signals to GATOR1 so as to modulate amino acid sensing by mTORC1. Second, how does activation of Src dissociate GATOR1 from the Rags? As the kinase activity of Src is involved in determining GATOR1 interaction with Rags, a possibility is that Src, directly or indirectly (via another kinase) phosphorylates some component(s) of GATOR1 and this event could be critical for Rag-GATOR1 complex formation. Alternatively, amino acid-mediated activation of Src could lead to the disruption of the GATOR1 complex itself.

The characterization of the mechanism by which Src dissociates GATOR1 from the Rags also warrants further investigation. Sustained activation of $\mathrm{mTORC1}$ is essential for cells to grow to their optimal size ${ }^{12}$, and our results indicate that Src regulates mTORC1-dependent cell growth as well as autophagy. This regulatory pathway might be disrupted in several human pathological conditions such as cancer and various metabolic disorders with mTORC1 involvement. Taken together, our study leads to the unexpected finding that Src is a crucial upstream regulator of mTORC1 that can sustain mTORC1 activity and promote cell survival under nutrient deprivation conditions, which might have a therapeutic potential in mTOR-associated pathologies.

\section{Methods}

Materials. Reagents were obtained from the following sources: DMEM/F12 (1:1) from life technologies (11320033); DMEM from hyclone (SH30081); dialyzed fetal bovine serum (dFBS) from GE Healthcare Life Sciences (SH30079); amino acids from Sigma Aldrich (A6282); Rapamycin from Sigma Aldrich (R0395); PP2 from Calbiochem (529573) and Dasatinib from LC Laboratories (D-3307). MK2206 was from Selleckchem and insulin was obtained from Sigma Aldrich.

Cell culture. SHSY-5Y cells (ATCC ${ }^{\circledR}$ CRL2266 ${ }^{\text {ma }}$ ) were grown in DMEM-F12 (1:1, Gibco) supplemented with $10 \%$ heat inactivated fetal bovine serum (FBS, Atlanta Biologicals), $2 \mathrm{mM}$ L-glutamine, $100 \mathrm{U} / \mathrm{ml}$ penicillin and $100 \mathrm{mg} / \mathrm{ml}$ streptomycin (Gibco) (referred as nutrient-rich media in manuscript). HEK293T, HeLa, and MEF cells were cultured in DMEM supplemented with $10 \%$ heat inactivated FBS, 2 $\mathrm{mM}$ L-glutamine, $100 \mathrm{U} / \mathrm{ml}$ penicillin, and $100 \mathrm{mg} / \mathrm{ml}$ streptomycin. FHC, Caco-2, and HT-29 cells were purchased from ATCC and cultured according to the manufacturer's protocol. HEK293T cells stably expressing Flag-raptor were a generous gift from Kartik Venkatachalam (UT Health Science Center). WT MEF cells were generated and cultured in our laboratory. $T s c 2^{-/-}$MEFs were kindly provided by Dr. Krymskaya (University of Pennsylvania).

Plasmids. For the generation of WT-Src-Flag construct, Src cDNA was amplified from pcDNA3 c-SRC (WT) (Addgene \#42202) kindly provided by Robert Lefkowitz and cloned into XbaI and HindIII sites of p3XFLAG-CMV-14 (Sigma). $\operatorname{Src} \Delta \mathrm{SH} 3$-Flag and $\operatorname{Src} \Delta \mathrm{SH} 2$-Flag constructs were generated using site-directed mutagenesis (Promega) with appropriate sets of primers. In brief, for the $\operatorname{Src} \Delta \mathrm{SH} 2$ Flag, we first inserted 2 PacI restrictions sites flanking the $\mathrm{SH} 2$ domain by using the following two primers pairs: 5prime_for: 5'-CCAGGCTGAGGAGTTTAATTAA GGCAAGATCACCA-3', 5prime_rev: 5' TGGTGATCTTGCCTTAATTAAACTC CTCAGCCTGG-3', 3prime_for: 5'-ACCACCGTGTGCCCCATTAATTAACCGC AGACTCAGGGC-3', and 3prime_rev: 5'- GCCCTGAGTCTGCGGTTAATTAAT
GGGGCACACGGTGGT-3'. After SH2 deletion, the construct was codon optimized using the following primers, 5'-ATCCAGGCTGAGGAGTGGTATTTTCC GCAGACTCAGGGC-3' and 5'- GCCCTGAGTCTGCGGAAAATACCACTCC TCAGCCTGGAT-3. For the Src $\Delta \mathrm{SH} 3$-Flag, we inserted a ApaI site at the end of the $\mathrm{SH} 2$ domain by using the following primers 5'- ATCCAGGCTGAGGGCC CGTATTTTGGCAAG-3' and 5'- CTTGCCAAAATACGGGCCCTCAGCCTGGA T-3'. The construct was then digested with ApaI and re-ligated without the SH3 domain. For the generation of CA-Src (Y530F-Src) we used the following primers 5'-GTCCACCGAGCCCCAGTTCCAGCCCGGGGA-3' and 5'-TCCCCGGGCTG GAACTGGGGCTCGGTGGAC-3'. The fllowing constructs were obtained from Addgene: Flag-HA-pcDNA3 (\# 10792), KD-Src (K295R-Src) (\#13659), HA-GSTRagA (\#19298), HA-GST-RagB (\#19301), HA-GST-RagC (\#19304), HA-GSTRagA $^{\text {GTP }}$ :Q66L (\#19300), HA-GST-RagC ${ }^{\text {GDP }}:$ S75L (\#19305), Flag-Depdc5 (\#46350), GFP-Depdc5 (\#46380), Flag-Nprl2 (\#46333), and HA-Nprl3 (\#46330). All constructs were sequence varified. GFP-LC3 and GFP-RFP-LC3 constructs were kindly provided by Ross Poche (Baylor College of Medicine).

Starvation protocol. For amino acid stimulation studies, sub-confluent cells were serum-starved for $16 \mathrm{~h}$ followed by amino acid starvation for $4 \mathrm{~h}$ prior to stimulation with a $10 \times$ mixture of total amino acids for $30 \mathrm{~min}$, unless otherwise indicated. The final concentration of amino acids used to stimulate the cells was $2 \times$. The 10× mixture of total amino acids was prepared from individual powders of amino acids 9,12 . For amino acid deprivation studies, complete growth medium was replaced by dialyzed serum for $4 \mathrm{~h}$. The recipe of the $10 \times$ mixture of total amino acids is provided in Supplementary Table 1. Chemical treatments were performed with indicated drugs for the last $2 \mathrm{~h}$ of amino acid starvation.

All the dry/powder amino acids were added to $500 \mathrm{ml}$ of $\mathrm{dH}_{2} \mathrm{O}$. Another $476 \mathrm{ml}$ of $\mathrm{dH}_{2} \mathrm{O}$ was added to make final volume 11 . Solution was mixed thoroughly by shaking and heating (mild) on a stir plate followed by filter sterilization using 0.2 micron filtration units.

Cell lysis and immunoblotting. After the end of treatment, cells were rinsed once with ice-cold PBS and harvested, and lysed using RIPA buffer $(50 \mathrm{mM}$ Tris- $\mathrm{HCl}$, ph 7.4, 1\% NP40, 0.5\% Na-deoxycholate, $0.1 \%$ SDS, $150 \mathrm{mM} \mathrm{NaCl}, 2 \mathrm{mM}$ EDTA and $50 \mathrm{mM} \mathrm{NaF}$ ) including a cocktail of protease (Roche, Basel, Switzerland) and phosphatase (MilliporeSigma, Billerica, MA) inhibitors. Protein concentration was measured with the bicinchoninic acid (BCA) protein assay kit (Pierce, Rockford, IL), using BSA as standard. Lysates were separated via SDS-PAGE and then transferred to polyvinyldifluoride (PVDF) membranes. Blots were incubated in blocking buffer $(5 \%, \mathrm{w} / \mathrm{v}$, dried skimmed milk in Tris-buffered saline, $\mathrm{pH} 7.4$, and $0.2 \%$ Tween 20 , TBST) followed by overnight incubation with appropriate antibodies diluted in blocking buffer. Blots were then exposed to the IRDye ${ }^{\circledast}$ secondary antibodies (LI-COR, Lincoln, NE) or HRP-conjugated antibodies diluted in TBST or blocking buffer, respectively, for $75 \mathrm{~min}$ at room temperature and washed again Blots were detected using appropriate image-developer. Uncropped scans of all blots are provided in Supplementary Fig. 6.

Immunofluorescence. Cells were grown on glass coverslips in 24-well plates. After the end of the treatment, cells were rinsed once with PBS and fixed with $1 \times$ PBS- $4 \%$ paraformaldehyde at room temperature (RT) for $15 \mathrm{~min}$. Cells were rinsed three times with PBS, permeabilized with $0.1 \%$ Triton X-100 in 1X PBS for $5 \mathrm{~min}$, rinsed twice with PBS, and blocked with blocking reagent $(0.1 \%$ saponin, $8 \%$ goat serum in PBS) for $1 \mathrm{~h}$ at RT. After one rinse with PBS, cells were incubated with primary antibody in blocking reagent overnight at $4{ }^{\circ} \mathrm{C}$ in a humidity chamber. After three PBS washes, coverslips were incubated with labeled secondary antibodies for $1 \mathrm{~h}$ at RT in the dark. After four PBS washes, coverslips were mounted with vectashield containing DAPI (H-1200) prior to microscopy. Images were acquired through $\times 63$ or $\times 40$ oil immersion objectives with either a Zeiss Axiotome fluorescence 
a

$$
\begin{aligned}
& -++ \text { GFP-Depdc5 } \\
& +- \text { - IP:IgG } \\
& \text { - + IP:Src } \\
& \text { Q }=\text { GFP-Depdc5 }
\end{aligned}
$$

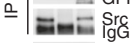

$$
\begin{aligned}
& ==\text { GFP-Depdc5 } \\
& \underset{\mathbb{W}}{9}-\cdots-\text { Sro } \\
& \text { د }--- \text { GAPDH }
\end{aligned}
$$

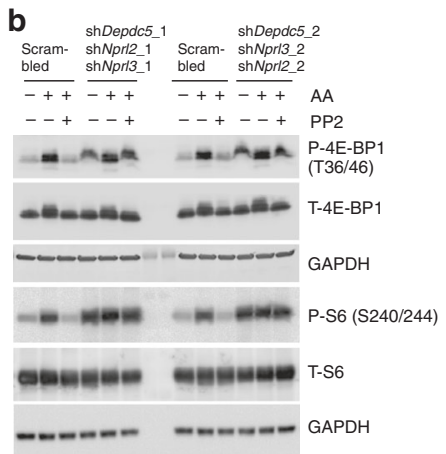

d

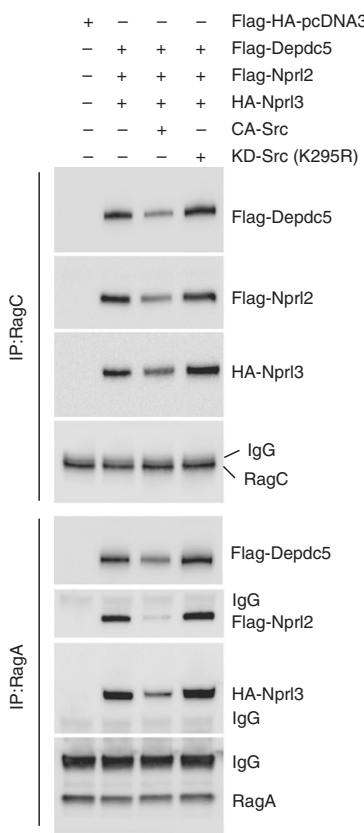

C

+ $\quad$ - Flag-HA-pcDNA3

- + + Flag-Depdc5

++ Flag-Nprl2

++ HA-Nprl3

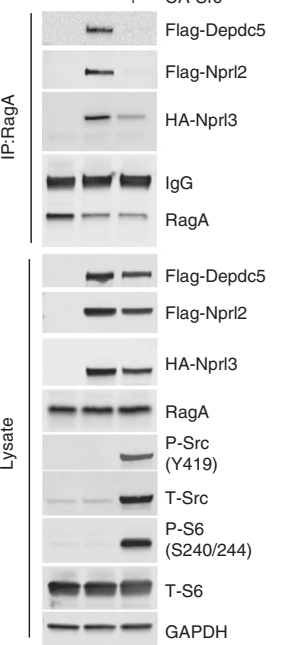

$+\quad$ - Flag-HA-pcDNA3

-++ Flag-Depdc5

-++ Flag-Npri2

++ HA-Nprl3

$$
\text { - + CA-Src }
$$

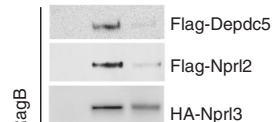

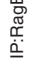

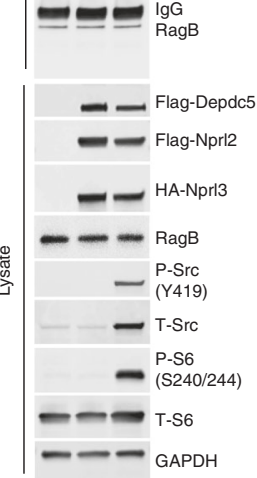

-

+ + - Flag-HA-pcDNA3

-++ Flag-Depdc5

-++ Flag-Nprl2

-++ HA-Nprl3

- + CA-SrC

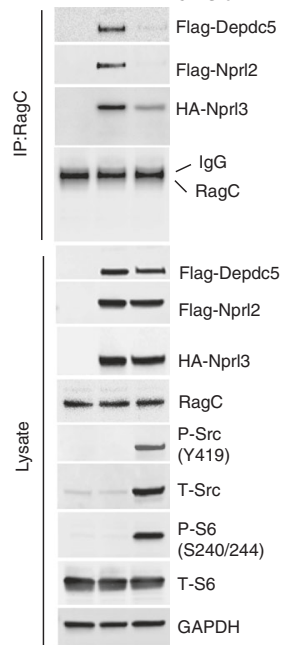

f

+ - - $\quad$ Flag-HA-pcDNA3

+++ Flag-Depdc5

-+++ Flag-Npri2

-+++ HA-Nprl3

-+++ WT-Src-Flag

- - + + PP2

-++ Flag-Depdc5

+++ Flag-Depdc5
-++ Flag-Nprl2

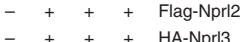

$-++\quad+$ CA-SrC

$-\quad+\quad+$ KD-Src (K295R)

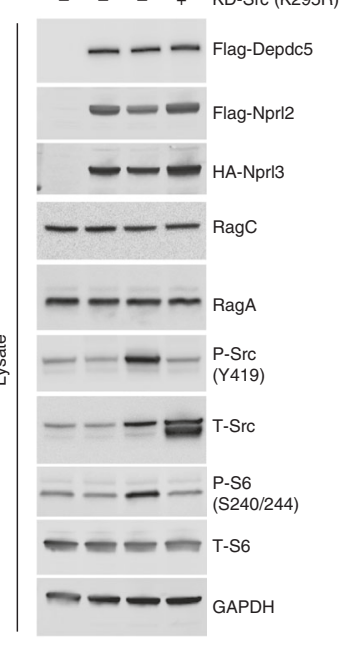

-++ Flag-Nprl2

-++ HA-Nprl3

- $~+\quad+$ WT-SrC
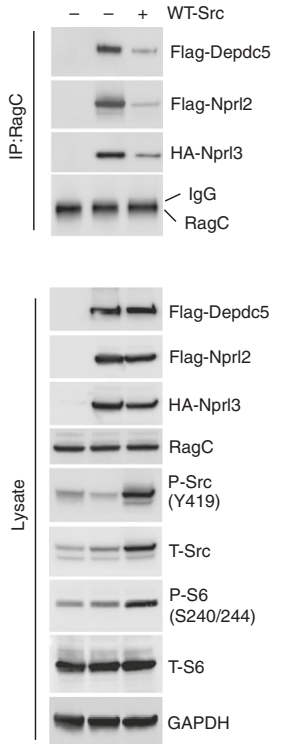
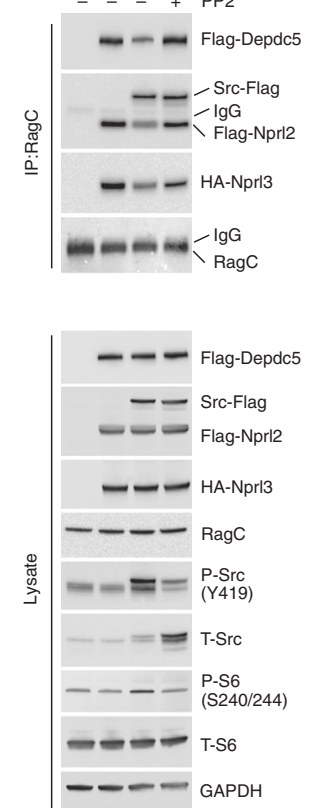

Fig. 4 Src kinase disrupts the interaction of GATOR1 with the Rags. a Src kinase interacts with the GATOR1 complex. HEK293 cells, transiently transfected with GFP-Depdc5, were lysed and subjected to immunoprecipation with antibodies against Src or IgG (negative control) followed by immunoblotting for the indicated proteins. Lysates were probed with antibodies as indicated. $\mathbf{b}$ HEK cells, transiently transfected with scramble shRNA or shRNAs targeting DEPDC5, NPRL2, and NPRL3 genes for $48 \mathrm{~h}$ prior to starvation and treatment with vehicle (DMSO) or PP2 for the last $2 \mathrm{~h}$ of starvation and then stimulated with amino acids (30 min). Immunoblot analyses were performed to measure the levels of the indicated proteins and phosphorylation states. $\mathbf{c}$ HEK293 cells, transiently transfected with the components of GATOR1 and constitutively active Src (CA-Src), were lysed and colP analyses were performed to test interaction of GATOR1 with RagA, RagB, or RagC. d HEK293 cells, transiently transfected with GATOR1 components, CA-Src or kinase-dead Src (K295R), were lysed and colP analyses were performed to test interaction of GATOR1 with RagA or RagC. e HEK293 cells, transiently transfected with the components of GATOR1 and wild-type Src (WT-Src), were lysed and subjected to RagC immunoprecipitation (IP) followed by immunoblotting for the indicated proteins. Lysates were probed with antibodies as indicated. f Inhibition of Src kinase promotes GATOR1-Rag interaction. HEK293 cells transfected as in e were treated with PP2 $(5 \mu \mathrm{M}, 24 \mathrm{~h})$ prior to lysis, and then subjected to RagC immunoprecipitation (IP) followed by immunoblotting for the indicated proteins. GAPDH was used as a loading control in all immunoblot assays 
a

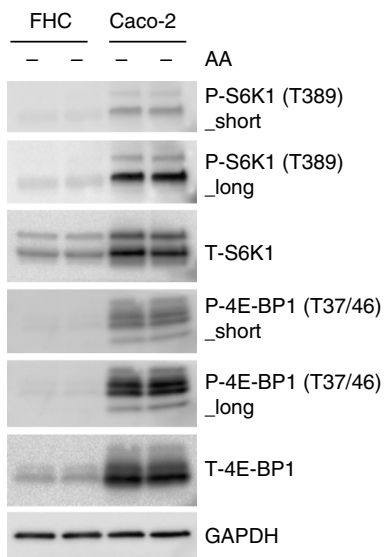

C

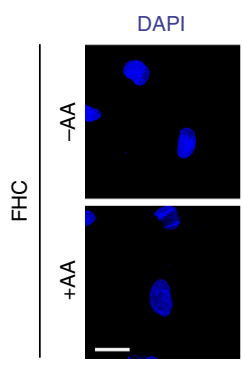

DAPI

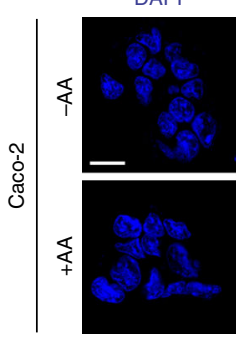

DAPI

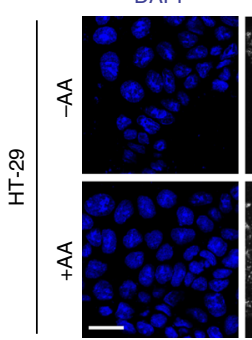

LAMP2

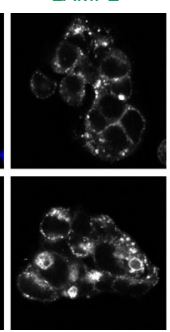

LAMP2

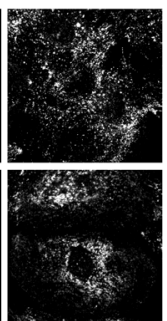

LAMP2

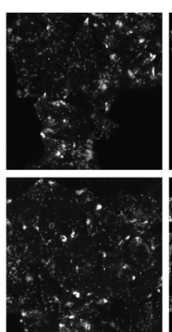

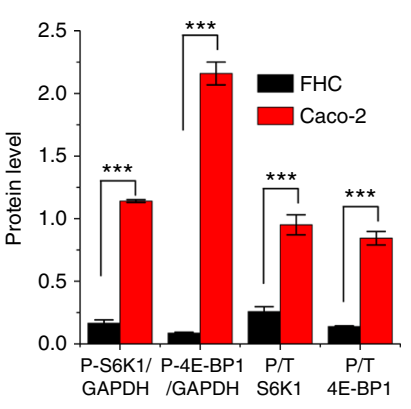
GAPDH /GAPDH S6K1 4E-BP1 b

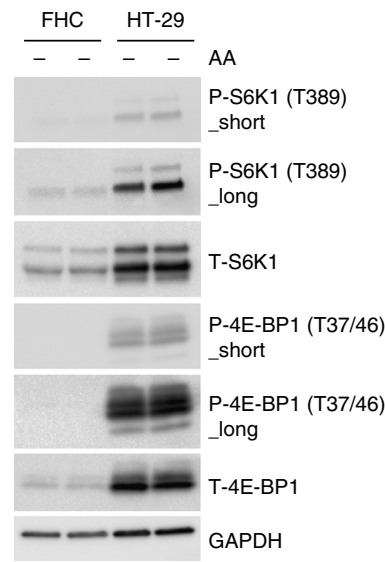

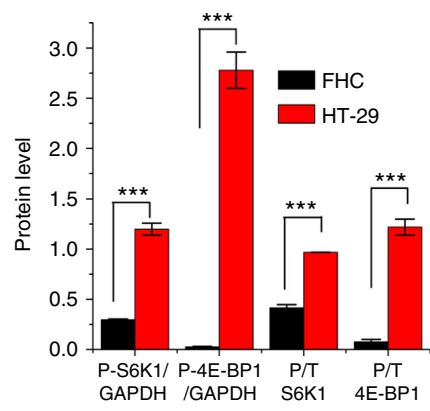
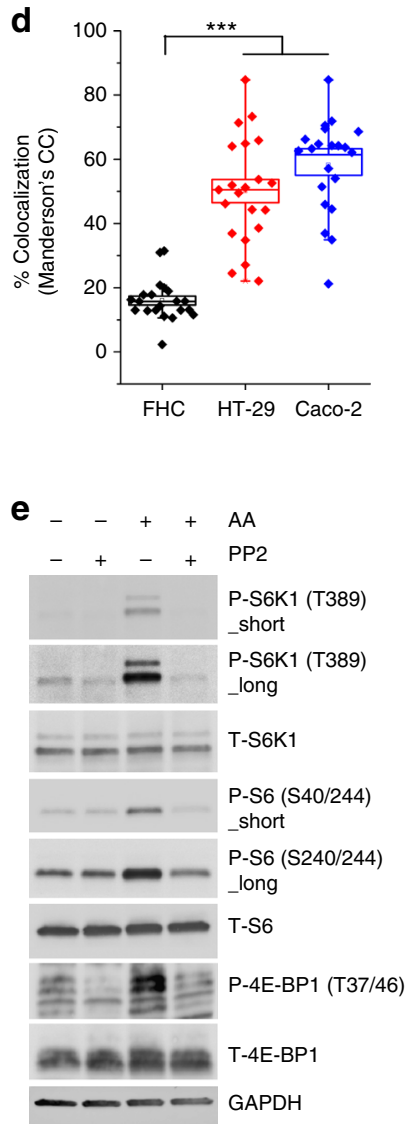

Fig. 5 Increased mTORC1 activity in cells with elevated Src activity. a FHC and Caco-2 cells were amino acid starved (kept in dialyzed serum) for $4 \mathrm{~h}$ prior to lysis and immunoblot analysis to measure the levels of the indicated proteins and phosphorylation states. b FHC and HT-29 cells were treated as in $\mathbf{a}$ prior to immunoblot analysis to measure the levels of the indicated proteins and phosphorylation states. Quantified data in $\mathbf{a}$ and $\mathbf{b}$ represent means \pm SEM of $n=2-3$ independent experiments. ${ }^{\star \star} p<0.001$. c FHC, Caco-2 and HT-29 cells were starved as in a and then stimulated with amino acids for 30 min prior to immunofluorescence labeling of endogenous LAMP2 (green) and mTOR (red). Bars indicate $40 \mu \mathrm{m}$ in FHC cells and $80 \mu \mathrm{m}$ in Caco-2 and HT29 cells. Representative cells are shown where yellow or orange pixels indicate co-localization in the merged images. Insets show selected fields that were magnified by a factor of 6 . Quantified data represent co-localization (Mander's coefficient) means of mTOR and LAMP2 in at least 15 cells for each condition. ${ }^{\star} p<0.05$ and ${ }^{\star *} p<0.01$. d Extent of co-localization of mTOR and LAMP2 in FHC, Caco-2 and HT-29 cells under amino acid starvation. Box plots in $\mathbf{c}$ and $\mathbf{d}$ represent quantified data of co-localization (Mander's coefficient) analysis in at least $n=30$ cells/line. ${ }^{* \star *} p<0.001$. e Caco-2 cells were amino acid starved (kept in dialyzed serum) for $4 \mathrm{~h}$ and treated with PP2 for the last $2 \mathrm{~h}$ prior to 30-min amino acid stimulation. Cells were lysed and immunoblot analyses were performed to measure the levels of the indicated proteins and phosphorylation states. Statistical differences between groups in a-d were determined using ANOVA with Tukey's post hoc test

microscope with Apotome feature engaged or a Zeiss 710 confocal laser microscope (Zeiss, Oberkochen, Germany). Representative images are shown in all figures at the same exposure and magnification. Antibody details are provided in Supplementary Table 2 .
Immunoprecipitation. For the immunoprecipitation analyses regulator components, cells were rinsed once with ice-cold PBS and lysed with ice-cold Chaps lysis buffer $(0.3 \%$ Chaps, $10 \mathrm{mM} \beta$-glycerol phosphate, $10 \mathrm{mM}$ pyrophosphate, $40 \mathrm{mM}$ Hepes $\mathrm{pH} 7.4,2.5 \mathrm{mM} \mathrm{MgCl}$, and 1 tablet of EDTA-free protease inhibitor 
a
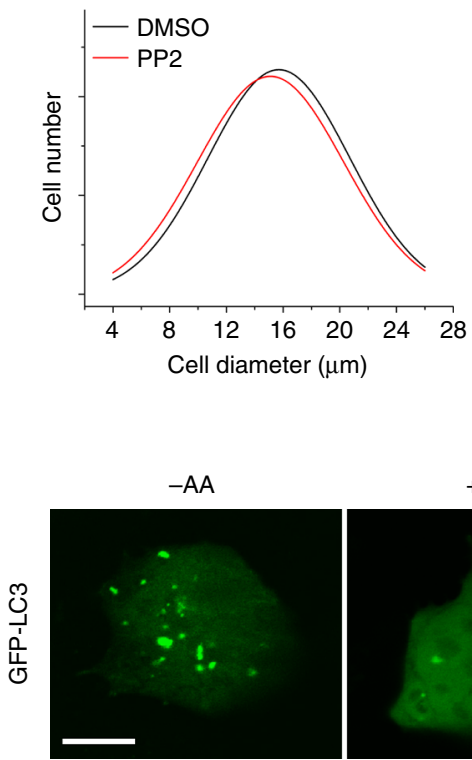

d
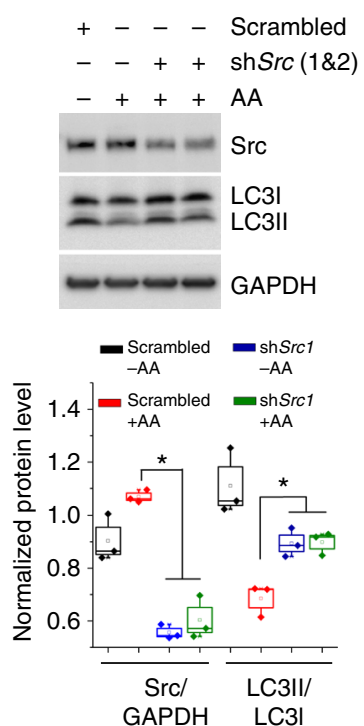

f
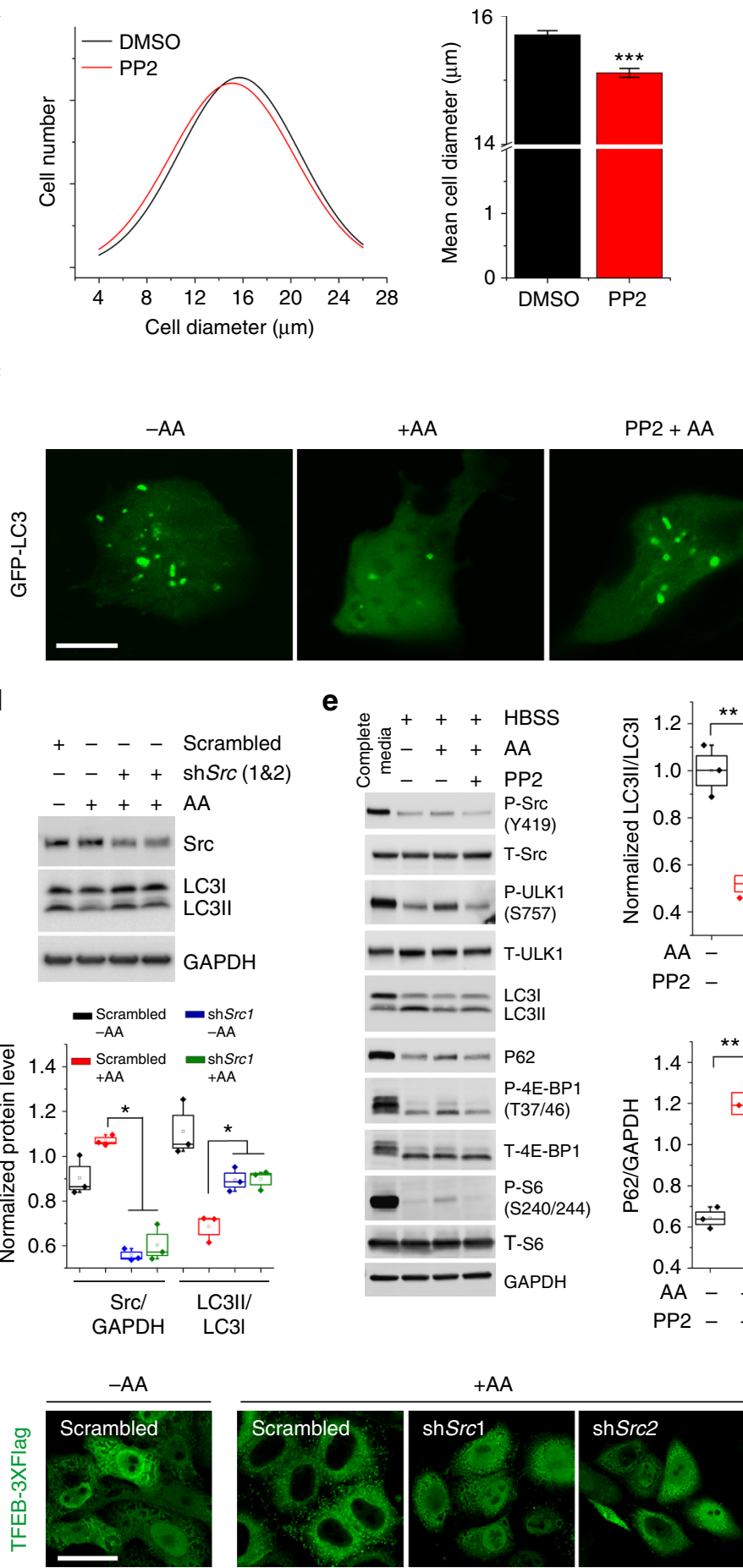

$+\mathrm{AA}$

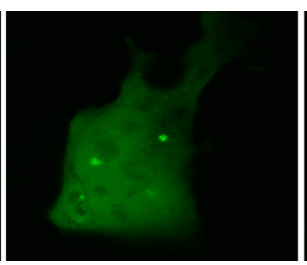

b

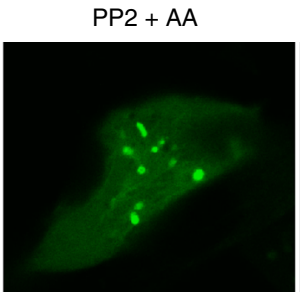

Rapamycin + AA
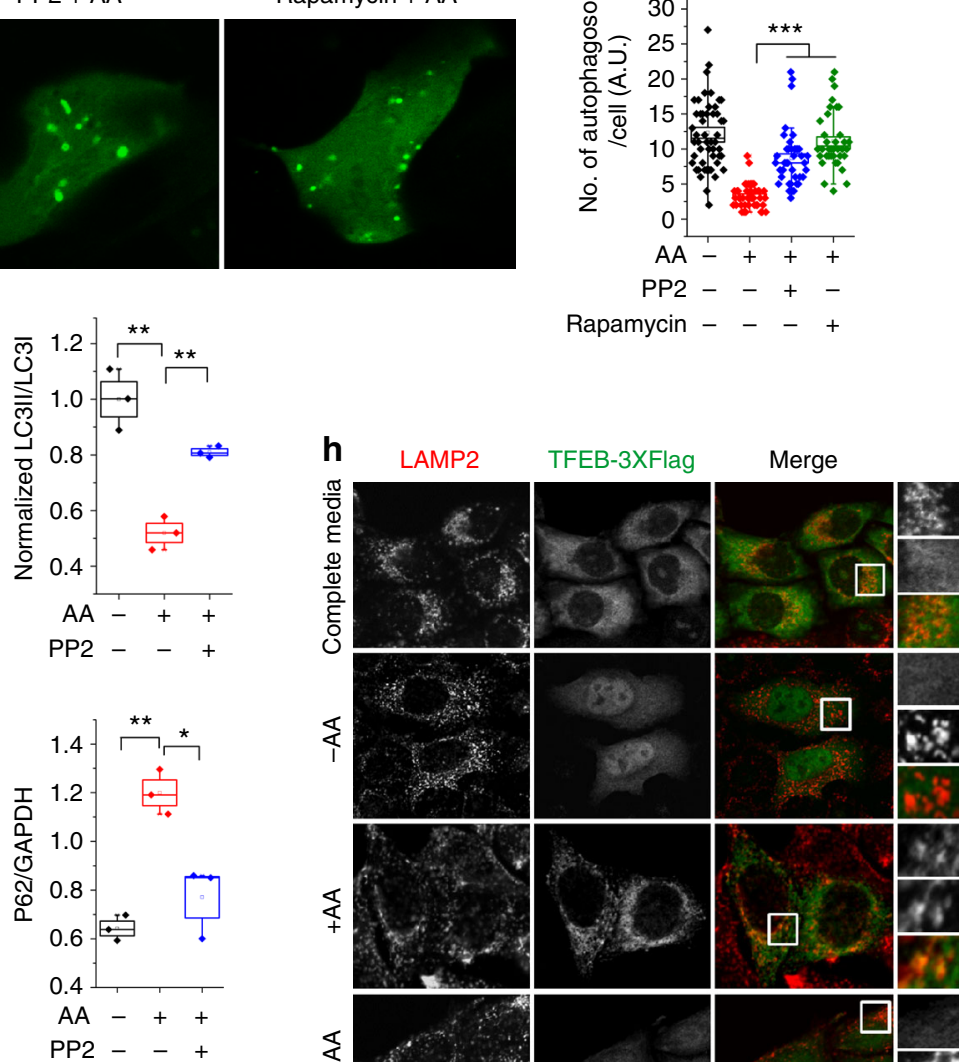

9
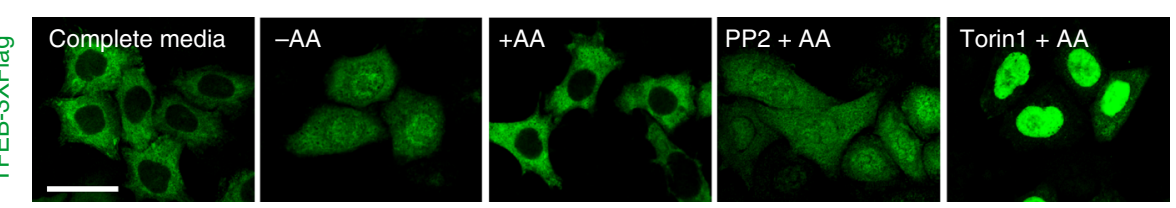

(Roche) per $25 \mathrm{ml}$ ). For all other immunoprecipitation assays, Cells were rinsed once with ice-cold PBS and lysed in ice-cold lysis buffer (40 mM HEPES [pH 7.4], $2 \mathrm{mM}$ EDTA, $10 \mathrm{mM}$ pyrophosphate, $10 \mathrm{mM}$ glycerophosphate, and $1 \%$ Triton $\mathrm{X}-100$, and one tablet of EDTA-free protease inhibitors (Roche) per $25 \mathrm{ml}$ ). The soluble fractions of cell lysates were isolated by centrifugation at $19,000 \times g$ for 15 min at $4{ }^{\circ} \mathrm{C}$. Protein concentration was measured with the BCA protein assay kit (Pierce, Rockford, IL). Primary antibodies were added to the lysates and incubated with rotation overnight at $4{ }^{\circ} \mathrm{C} .60 \mu \mathrm{l}$ of a $50 \%$ slurry of protein G-agarose beads (Roche, Basel, Switzerland) was added and incubation continued for $4 \mathrm{~h}$ at $4^{\circ} \mathrm{C}$. Immunoprecipitates were washed three times $\left(7 \mathrm{~min}\right.$ each at $\left.4^{\circ} \mathrm{C}\right)$ with lysis buffer containing $150 \mathrm{mM} \mathrm{NaCl}$. Immunoprecipitated proteins were denatured by the addition of 20-30 $\mu$ l of sample buffer (including 2-mercaptoethanol) and boiling for $5 \mathrm{~min}$, resolved by $4-20 \%$ SDS-PAGE (Initrogen, Carlsbad, CA), and analyzed by immunoblotting. For co-transfection experiments, $1.5 \times 10^{6}$ cells were plated in 
Fig. 6 Src regulates cell growth, TFEB localization and autophagy. $\mathbf{a}, \mathbf{b}$ The histogram shows the cell size of SH-SY5Y (a) and MEF (b) cells treated with DMSO or PP2 $(5 \mu \mathrm{M}$ for $24 \mathrm{~h})$. The bar diagram represents the mean diameter of at least $10^{4}$ cells. Error bars represent values in \pm SEM. ${ }^{\star \star \star} p<1 \times 10^{-6}$. c SH-SY5Y cells expressing GFP-LC3 were starved and treated with vehicle (DMSO), PP2 (10 $\mu \mathrm{M})$, or rapamycin $(600 \mathrm{nM})$ for the last $2 \mathrm{~h}$ of starvation and then stimulated with amino acids $\left(30 \mathrm{~min}\right.$ ). Box plots represent quantified data of lipidated-LC3 puncta from at least 35 cells. ${ }^{\star} p<0.05$ and ${ }^{\star \star \star} p<$ 0.001. Bar indicates $20 \mu \mathrm{m}$. d Immunoblot analysis of SH-SY5Y cells transiently transfected with scrambled shRNA or shSrc prior to starvation and amino acid stimulation (30 min). e Immunoblot analysis of SH-SY5Y cells left in growth media or starved and treated with vehicle (DMSO) or PP2 (10 $\mu$ M) for the last $2 \mathrm{~h}$ of starvation and then stimulated with amino acids (30 min). Box plots in $\mathbf{d}$ and e represent SE of $n=3$ independent experiments. ${ }^{\star} p<0.05$ and ${ }^{\star \star} p$ $<0.01$. $\mathbf{f e L a}$ cells, stably transfected with Flag-TFEB, were transiently transfected with scrambled or shSrc and treated as in $\mathbf{d}$. Cells were immunostained with Flag (green) for the analyses of cytosolic and nuclear localization of TFEB. Bar indicates $80 \mu \mathrm{m} . \mathbf{g}$ HeLa cells, stably transfected with Flag-TFEB, were treated as in e. Torin1 ( $300 \mathrm{nM}$ for $2 \mathrm{~h}$ ) was used as a control (mTOR inhibitor). Cells were immunostained with Flag (green) for the analyses of cytosolic and nuclear localization of TFEB. Bar indicates $40 \mu \mathrm{m}$. h HeLa cells, stably transfected with Flag-TFEB, were treated as in e prior to immunofluorescence labeling of LAMP2 (red) and Flag (green). Representative cells are shown where yellow or orange pixels indicate co-localization in the merged images. In all images, insets show selected fields that were magnified four times and their overlays. Bar indicates $30 \mu \mathrm{m}$. GAPDH was used as a loading control in all immunoblot assays. Statistical differences between groups in a-e were determined using ANOVA with Tukey's post hoc test
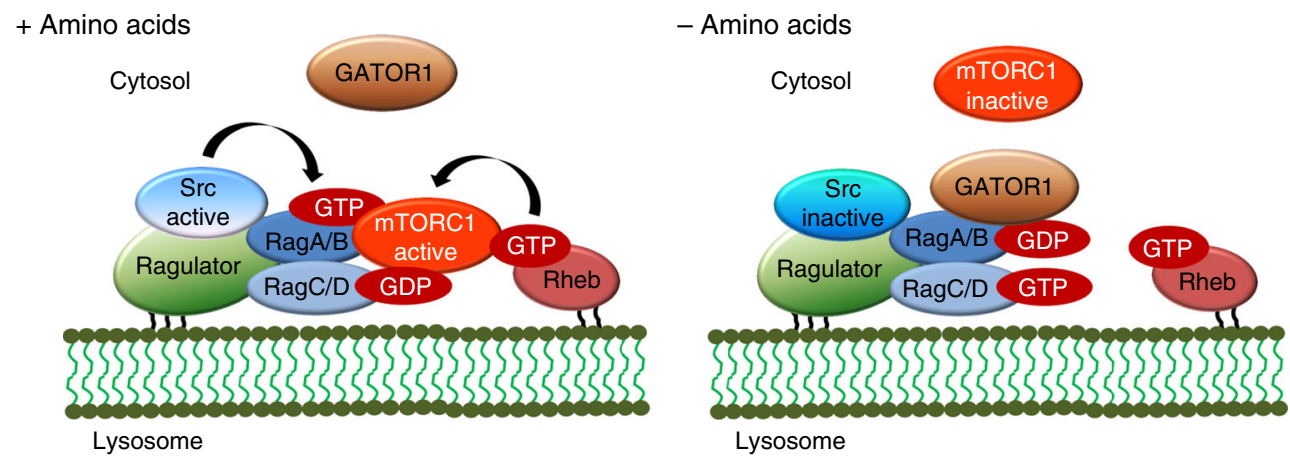

Fig. 7 Schematic diagram for Src-dependent regulation of mTORC1. The model shows that Src kinase regulates amino acid-mediated mTORC1 activity by controlling GATOR1-Rag interaction

$10 \mathrm{~cm}$ culture dishes. Twenty hours later, cells were transfected with the appropriate expression plasmids indicated in the figures in equal amounts. The total amount of plasmid DNA in each transfection was normalized to $5 \mu \mathrm{g}$ with empty vector. Thirty-six hours after transfection, cells were lysed as described above.

Cytosolic/light membrane and heavy membrane fractions. SH-SY5Y cells were grown in two $15-\mathrm{cm}$ dishes per condition. Cells (90-95\% confluent) were rinsed with ice-cold PBS, scraped into ice-cold PBS, pelleted by centrifugation at $1000 \times g$ for $2 \mathrm{~min}$ at $4^{\circ} \mathrm{C}$. Supernatants were discarded and pellets were resuspended in $350 \mu \mathrm{l}$ cold hypotonic lysis buffer (10 mM HEPES, pH 7.2, $10 \mathrm{mM} \mathrm{KCl}, 2 \mathrm{mM}$ $\mathrm{MgCl} 2,20 \mathrm{mM} \mathrm{NaF}, 100 \mu \mathrm{M}$ sodium orthovanadate, $250 \mathrm{mM}$ sucrose with freshly added protease, and phosphatase inhibitors). Cells were mixed thoroughly by vortexing. Cells were mechanically lysed by drawing five times through a $23 \mathrm{G}$ needle and centrifuged at $500 \times g$ for $10 \mathrm{~min}$ at $4{ }^{\circ} \mathrm{C}$, yielding a post-nuclear supernatant (PNS). PNS was used for the fractionation of light membrane/cytosolic and heavy membrane fractions. The PNS was centrifuged at $20,000 \times g$ for $2 \mathrm{~h}$ to separate the soluble supernatant (cytosolic/light membrane fraction) from the insoluble pellet (heavy membrane fraction). The pellet was lysed in RIPA buffer (50 mM Tris-HCl, ph 7.4, 1\% NP40, 0.5\% Na-deoxycholate, 0.1\% SDS, $150 \mathrm{mM}$ $\mathrm{NaCl}, 2 \mathrm{mM}$ EDTA, and $50 \mathrm{mM} \mathrm{NaF}$ ) including a cocktail of protease (Roche) and phosphatase (MilliporeSigma) inhibitors. Protein concentration was measured with the BCA protein assay kit (Promega) using BSA as standard. Proteins were denatured by the addition of 20-30 $\mu$ of sample buffer (including 2-mercaptoethanol) and boiling for $10 \mathrm{~min}$, resolved by $4-20 \%$ SDS-PAGE (Invitrogen), and analyzed by immunoblotting.

Separation of lysosome-enriched fraction. Lysosomes were enriched from SHSY5Y cells from three near-confluent $15-\mathrm{cm}$ dishes. Briefly, cells where washed with ice-cold PBS, pelleted at $500 \times g$ for $10 \mathrm{~min}$ in assay buffer $(0.25 \mathrm{M}$ sucrose, $\mathrm{pH}$ 7.2) and homogenized using a Dounce homogenizer. The homogenate was centrifuged at $800 \times g$ for $10 \mathrm{~min}$, the supernatant was collected separately, and the pellet was rinsed with assay buffer followed by another centrifugation at $800 \times g$ for $10 \mathrm{~min}$. The supernatants from both centrifugations were combined, mixed thoroughly with assay buffer and Percoll (20\% final concentration) by vortexing. Samples were centrifuged at $36,000 \times g$ for $30 \mathrm{~min}$ using SW 40 Ti rotor (Beckman). A total of 10 fractions were collected. Fractions were diluted in 5-10 volumes of assay buffer and centrifuged at $36,000 \times g$ for $15 \mathrm{~min}$. Pellets were then resuspended in $200 \mu \mathrm{l}$ of RIPA buffer. These samples were then analyzed by immunoblotting assay as described earlier.

Cell size determination. SH-SY5Y, MEF, and HEK293T cells were cultured as described earlier. Cells, $70-80 \%$ confluent, were then treated with DMSO or $5 \mu \mathrm{M}$ PP2 for $24 \mathrm{~h}$. After the treatment, cells were washed with $1 \times$ PBS twice, trypsinized and resuspended to a concentration of $1 \times 10^{5}$ cells per $\mathrm{ml}$ in $1 \times \mathrm{PBS}$. Cell number and size of each condition was measured using the size histogram function of the Cellometer Auto 1000 (Nexcelom Bioscience, USA). Line-plot profile was created from binned-histogram generated from a total of at least $1 \times 10^{4}$ cells per condition using Origin Pro software (OriginLab Corporation, Northhampton, MA).

shRNA knockdown. shRNA-mediated knockdowns were performed using jetPRIME transfection reagent (Polyplus, Illkirch France). Cells were transfected according to the manufacturer's protocol and processed for the given experiments at 70-72 $\mathrm{h}$ post-transfection. SH-SY5Y cells transfected with shRNA-Src or scrambled (ORIGENE, Rockville, MD) were maintained in DMEM-F12/10\% FBS. For the amino acid-stimulation experiments, $50 \mathrm{~h}$ after transfection cells were starved of serum $(16 \mathrm{~h})$ and amino acids (last $4 \mathrm{~h}$ ) followed by stimulation with amino acids $(30 \mathrm{~min})$. shRNA-mediated knockdowns of GATOR1 genes were performed in HEK cells using jetPRIME transfection reagent (Polyplus, Illkirch France). Cells were transfected according to the manufacturer's protocol and processed for the given experiments $48 \mathrm{~h}$ post-transfection. For the amino acidstimulation experiments, $48 \mathrm{~h}$ after transfection the cells were starved of serum (16 $\mathrm{h}$ ) and amino acids (last $4 \mathrm{~h}$ ) followed by $2 \mathrm{~h}$ of PP2 treatment (last $2 \mathrm{~h}$ of amino acid starvation) prior to stimulation with amino acids $(30 \mathrm{~min})$.

The following Src shRNAs were used:

shSrc1: 5'-ATGACCTTATGTGCCAGTGCTGGCGGAAG-3';

shSrc2: 5'-CAGATCGCTTCAGGCATGGCCTATGTGGA-3';

shSrc3: 5'-CTGGAGGCAATCAAGCAGACATAGAAGAG-3'

Scrambled: Non-effective 29-mer scrambled shRNA cassette (OriGene, TR30015)

shRNAs targeting components of GATOR1 complex were purchased from CellBased Assay Screening Service (C-BASS) in Baylor College of Medicine. The following shRNAs were used:

Depdc5 1: 5'-AGGTCAAGTCTCTTAAGGA-3'

Depdc5_2: 5'-AGGAGTGTGCACAGATGTA-3'

Nprl2_1: 5'-CCTGTCTTTACCAAAGACA-3' 
Nprl2 2: 5'-CGGGATGTGTTCCAGCTAT-3' Nprl3_1: 5'-GTGATAAACTGTCTGCATA-3' Nprl3_2: 5'-ATGTTATTCTGGCAACAAT-3'

Quantitative real-time PCR. Total RNA was extracted from HEK cells transiently transfected with scramble shRNA or shRNAs targeting DEPDC5, NPRL2 and $N P R L 3$ genes for $48 \mathrm{~h}$. RNA was isolated using the RNEasy kit (Qiagen) according to the manufacturer's instructions. RNA quantification was performed using the Nano-Drop 8000 (Thermo Fischer) followed by cDNA synthesis using QuantiTect Reverse Transcription kit (Qiagen). Quantitative real-time PCR was performed by using iQ SYBR Green Supermix (Bio-rad, cat\# 172-5124) on the CFX96 Touch Real-Time Detection System (Bio-Rad Laboratories). Analyses were conducted using CFX manager software (Bio-Rad) and the threshold cycle (CT) was extracted from the PCR amplification plot. The change in messenger RNA levels was expressed as fold change. The primers used for qRT-PCR are indicated below.

NPRL2_Forward: TGGGACCCAAGATCACCTAT

NPRL2 Reverse: GCTTGTTCTGCAGCTCTGG

NPRL3_Forward: GGGCAGATCTCCAAAACAGA

NPRL3_Reverse: ACGGGACAGGTTATGCAGAC

DEPDC5 Forward: TAACCCACAGTTCACCAGCC

DEPDC5_Reverse: GGGGATATGTGGCGACTAAA

Statistics. Bar diagrams are reported as mean \pm SEM and Box plots are reported as SE, unless otherwise specified. Statistical differences between groups were determined using ANOVA with Tukey's post hoc test. Statistical analysis was performed in Origin Pro (OriginLab Corporation, Northhampton, MA) with a significance level of ${ }^{*} p<0.05,{ }^{* *} p<0.01$, and ${ }^{* *} p<0.001$.

\section{Data availability}

The data that support the findings of this study are available from the authors on reasonable request.

Received: 26 February 2018 Accepted: 18 September 2018

Published online: 19 October 2018

\section{References}

1. Hsu, P. P. et al. The mTOR-regulated phosphoproteome reveals a mechanism of mTORC1-mediated inhibition of growth factor signaling. Science 332, 1317-1322 (2011).

2. Menon, S. et al. Spatial control of the TSC complex integrates insulin and nutrient regulation of mTORC1 at the lysosome. Cell 156, 771-785 (2014)

3. Rebsamen, M. et al. SLC38A9 is a component of the lysosomal amino acidsensing machinery that controls mTORC1. Nature 519, 477-481 (2015).

4. Kim, D.-H. et al. mTOR interacts with raptor to form a nutrient-sensitive complex that signals to the cell growth machinery. Cell 110, 163-175 (2002).

5. Ma, X. M. \& Blenis, J. Molecular mechanisms of mTOR-mediated translational control. Nat. Rev. Mol. Cell Biol. 10, 307-318 (2009).

6. Zhang, Y. et al. Coordinated regulation of protein synthesis and degradation by mTORC1. Nature 513, 440-443 (2014).

7. Mizushima, N., Yoshimorim, T. \& Levine, B. Methods in mammalian autophagy research. Cell 140, 313-326 (2010).

8. Nicklin, P. et al. Bidirectional transport of amino acids regulates $\mathrm{mTOR}$ and autophagy. Cell 136, 521-534 (2009).

9. Bar-Peled, L. et al. A tumor suppressor complex with GAP activity for the Rag GTPases that signal amino acid sufficiency to mTORC1. Science 340, 1100-1106 (2013).

10. Bar-Peled, L., Schweitzer, L. D., Zoncu, R. \& Sabatini, D. M. An expanded Ragulator is a GEF for the Rag GTPases that signal amino acid levels to mTORC1. Cell 150, 1196-1208 (2012).

11. Zoncu, R. et al. mTORC1 senses lysosomal amino acids through an inside-out mechanism that requires the Vacuolar $\mathrm{H}(+)$-ATPase. Science 334, 678-683 (2011).

12. Sancak, Y. et al. The Rag GTPases bind raptor and mediate amino acid signaling to mTORC1. Science 320, 1496-1501 (2008).

13. Sancak, Y. et al. Ragulator-Rag complex targets mTORC1 to the lysosomal surface and is necessary for its activation by amino acids. Cell 141, 290-303 (2010).

14. Chantranupong, L. et al. The Sestrins interact with GATOR2 to negatively regulate the amino acid sensing pathway upstream of mTORC1. Cell Rep. 9, 1-8 (2014)

15. Parmigiani, A. et al. Sestrins inhibit mTORC1 kinase activation through the GATOR complex. Cell Rep. 9, 1281-1291 (2014).

16. Corradetti, M. N. \& Guan, K. L. Upstream of the mammalian target of rapamycin: do all roads pass through mTOR? Oncogene 25, 6347-6360 (2006).
17. Menon, S. \& Manning, B. D. Common corruption of the mTOR signaling network in human tumors. Oncogene 27, S43-S51 (2008).

18. Irby, R. B. \& Yeatman, T. J. Role of Src expression and activation in human cancer. Oncogene 19, 5636 (2000).

19. Roskoski, R. Jr Src protein-tyrosine kinase structure and regulation. Biochem. Biophys. Res. Commun. 324, 1155-1164 (2004).

20. Haura, E. B. et al. Phase I/II study of the Src inhibitor dasatinib in combination with erlotinib in advanced non-small-cell lung cancer. J. Clin. Oncol. 28, 1387-1394 (2010).

21. Pal, R. et al. Src-dependent impairment of autophagy by oxidative stress in a mouse model of Duchenne muscular dystrophy. Nat. Commun. 5, 4425-4425 (2014).

22. Xu, W., Harrison, S. C. \& Eck, M. J. Three-dimensional structure of the tyrosine kinase c-Src. Nature 385, 595-602 (1997).

23. Okada, M., Howell, B. W., Broome, M. A. \& Cooper, J. A. Deletion of the SH3 domain of Src interferes with regulation by the phosphorylated carboxylterminal tyrosine. J. Biol. Chem. 268, 18070-18075 (1993).

24. Kmiecik, T. E. \& Shalloway, D. Activation and suppression of pp60c-src transforming ability by mutation of its primary sites of tyrosine phosphorylation. Cell 49, 65-73 (1987).

25. Piwnica-Worms, H., Saunders, K. B., Roberts, T. M., Smith, A. E. \& Cheng, S $\mathrm{H}$. Tyrosine phosphorylation regulates the biochemical and biological properties of pp60c-src. Cell 49, 75-82 (1987).

26. Palmieri, M. et al. mTORC1-independent TFEB activation via Akt inhibition promotes cellular clearance in neurodegenerative storage diseases. Nat. Commun. 8, 14338 (2017).

27. Demetriades, C., Doumpas, N. \& Teleman, A. A. Regulation of TORC1 in response to amino acid starvation via lysosomal recruitment of TSC2. Cell 156, 786-799 (2014).

28. Zhu, S., Bjorge, J. D. \& Fujita, D. J. PTP1B contributes to the oncogenic properties of colon cancer cells through Src activation. Cancer Res. 67, 10129 (2007).

29. Gómez, S. et al. Independent regulation of adherens and tight junctions by tyrosine phosphorylation in Caco-2 cells. Biochim. Biophys. Acta 1452, 121-132 (1999).

30. Basuroy, S. et al. Expression of kinase-inactive c-Src delays oxidative stressinduced disassembly and accelerates calcium-mediated reassembly of tight junctions in the Caco-2 cell monolayer. J. Biol. Chem. 278, 11916-11924 (2003).

31. Basu, N., Bhandari, R., Natarajan, V. T. \& Visweswariah, S. S. Cross talk between receptor guanylyl cyclase $\mathrm{C}$ and c-Src tyrosine kinase regulates colon cancer cell cytostasis. Mol. Cell Biol. 29, 5277-5289 (2009).

32. Durán, RaúlV. et al. Glutaminolysis activates Rag-mTORC1 signaling. Mol. Cell 47, 349-358 (2012).

33. Mizushima, N. Methods for monitoring autophagy using GFP-LC3 transgenic mice. Methods Enzymol. 452, 13-23 (Academic Press, Cambridge, MA, 2009).

34. Kang, S. A. et al. mTORC1 phosphorylation sites encode their sensitivity to starvation and rapamycin. Science 341, 1236566-1236566 (2013).

35. Zhou, C. et al. Monitoring autophagic flux by an improved tandem fluorescent-tagged LC3 (mTagRFP-mWasabi-LC3) reveals that high-dose rapamycin impairs autophagic flux in cancer cells. Autophagy 8, 1215-1226 (2012).

36. Lee, J. M. et al. Nutrient sensing nuclear receptors coordinate autophagy. Nature 516, 112-115 (2014)

37. Sardiello, M. Transcription factor EB: from master coordinator of lysosomal pathways to candidate therapeutic target in degenerative storage diseases. Ann. N. Y. Acad. Sci. 1371, 3-14 (2016)

38. Sardiello, M. et al. A gene network regulating lysosomal biogenesis and function. Science 325, 473 (2009).

39. Settembre, C. et al. A lysosome-to-nucleus signalling mechanism senses and regulates the lysosome via mTOR and TFEB. EMBO J 31, 1095-1108 (2012).

40. Roczniak-Ferguson, A. et al. The transcription factor TFEB links mTORC1 signaling to transcriptional control of lysosome homeostasis. Sci. Signal 5, ra42 (2012).

41. Martina, J. A., Chen, Y., Gucek, M. \& Puertollano, R. MTORC1 functions as a transcriptional regulator of autophagy by preventing nuclear transport of TFEB. Autophagy 8, 903-914 (2012).

42. Martina, J. A. \& Puertollano, R. Rag GTPases mediate amino acid-dependent recruitment of TFEB and MITF to lysosomes. J. Cell Biol. 200, 475-491 (2013).

43. Weichhart, T., Hengstschlager, M. \& Linke, M. Regulation of innate immune cell function by mTOR. Nat. Rev. Immunol. 15, 599-614 (2015).

44. Chantranupong, L. et al. The CASTOR proteins are arginine sensors for the mTORC1 pathway. Cell 165, 153-164 (2016).

45. Saxton, R. A. et al. Structural basis for leucine sensing by the Sestrin2mTORC1 pathway. Science 351, 53-58 (2016).

46. Wolfson, R. L. et al. Sestrin2 is a leucine sensor for the mTORC1 pathway. Science 351, 43-48 (2016). 
47. Sütterlin, C., Polishchuk, R., Pecot, M. \& Malhotra, V. The Golgi-associated protein GRASP65 regulates spindle dynamics and is essential for cell division. Mol. Biol. Cell 16, 3211-3222 (2005).

48. Laboissière, M. C. A., Sturley, S. L. \& Raines, R. T. The essential function of protein-disulfide isomerase is to unscramble non-native disulfide bonds. J. Biol. Chem. 270, 28006-28009 (1995).

\section{Acknowledgements}

We thank Vera P. Krymskaya for the Tsc2 mutant MEFs. We thank David Reiner and Kartik Venkatachalam for critical reading of the manuscript. This work was supported by NIH grant NS079618 to M.S., grants from the Beyond Batten Disease Foundation and Charlotte \& Gwenyth Gray Foundation to M.S., and grants from The National Institute of Arthritis and Musculoskeletal and Skin Diseases of the National Institutes of Health under Award Number R01 AR061370 to G.G.R.

\section{Author contributions}

R.P. and M.S. conceived the project and designed the experiments. R.P., M.P., A.C., T.K. and A.D. performed experiments and analyzed the data under the supervision of J.R.N, G.G.R. and M.S., R.P. and M.S. wrote the manuscript with help from G.G.R.

\section{Additional information}

Supplementary Information accompanies this paper at https://doi.org/10.1038/s41467018-06844-4.

\section{Competing interests: The authors declare no competing interests.}

Reprints and permission information is available online at http://npg.nature.com/ reprintsandpermissions/

Publisher's note: Springer Nature remains neutral with regard to jurisdictional claims in published maps and institutional affiliations.

\section{(c) (i)}

Open Access This article is licensed under a Creative Commons Attribution 4.0 International License, which permits use, sharing, adaptation, distribution and reproduction in any medium or format, as long as you give appropriate credit to the original author(s) and the source, provide a link to the Creative Commons license, and indicate if changes were made. The images or other third party material in this article are included in the article's Creative Commons license, unless indicated otherwise in a credit line to the material. If material is not included in the article's Creative Commons license and your intended use is not permitted by statutory regulation or exceeds the permitted use, you will need to obtain permission directly from the copyright holder. To view a copy of this license, visit http://creativecommons.org/ licenses/by/4.0/.

(C) The Author(s) 2018 